\title{
Canonical phase space approach to the noisy Burgers equation: Probability distributions
}

\author{
Hans C. Fogedby \\ * Institute of Physics and Astronomy, University of Aarhus, DK-8000, Aarhus C, Denmark \\ and \\ NORDITA, Blegdamsvej 17, DK-2100, Copenhagen Ø, Denmark
}

\begin{abstract}
We present a canonical phase space approach to stochastic systems described by Langevin equations driven by white noise. Mapping the associated Fokker-Planck equation to a Hamilton-Jacobi equation in the nonperturbative weak noise limit we invoke a principle of least action for the determination of the probability distributions. We apply the scheme to the noisy Burgers and KPZ equations and discuss the time-dependent and stationary probability distributions. In one dimension we derive the long-time skew distribution approaching the symmetric stationary Gaussian distribution. In the short-time region we discuss heuristically the nonlinear soliton contributions and derive an expression for the distribution in accordance with the directed polymer-replica and asymmetric exclusion model results. We also comment on the distribution in higher dimensions.
\end{abstract}

PACS numbers: 05.10.Gg, 05.45.-a, 64.60.Ht, 05.45.Yv

\section{INTRODUCTION}

This is the third of a series of papers where we investigate the noisy Burgers equation in the context of modelling a growing interface; for a brief account of the present work we also we refer to [1]. In the previous two papers, in the following denoted paper I [2] and paper II [3]; a brief account of paper II also appeared in 何, we discussed the originally proposed one dimensional noiseless Burgers equation [5,6] from a solitonic point of view and the noisy one dimensional Burgers equation [7. 8 in terms of a Martin-Siggia-Rose path integral [9 14], respectively.

Phenomena far from equilibrium are widespread, including turbulence in fluids, interface and growth problems, chemical reactions, biological systems, and even economical and sociological structures. In recent years much of the focus of modern statistical physics and soft condensed matter has shifted towards such systems. Drawing on the case of static and dynamic critical phenomena in and close to equilibrium 15, 16], where scaling, critical exponents, and universality have served to organize our understanding and to provide calculational tools, a similar approach has been advanced towards nonequilibrium phenomena with the purpose of elucidating scaling properties and more generally the morphology or pattern formation in a driven state.

In this context the noisy Burgers equation provides maybe the simplest continuum description of an open driven nonlinear system exhibiting scaling and pattern formation. The Burgers equation was originally suggested in the one dimensional noiseless version [5, 6, 17,19

$$
\left(\frac{\partial}{\partial t}-\lambda u \nabla\right) u=\nu \nabla^{2} u
$$

as a model for irrotational hydrodynamical fluid flow. Here $u$ is the irrotational velocity field and $\nu$ a damping constant or viscosity. Choosing the nonlinear coupling $\lambda=-1$, we recognize the usual nonlinear convective term 20]. As a nonlinear model for hydrodynamical turbulence [21 27] Eq. (1.1) has been studied intensively. It was early recognized that the nonlinear structure of the damped velocity field is dominated by shock waves [6, 19], yielding an inverse cascade and that the Burgers equation does in fact not characterize Navier-Stokes turbulence which is governed by a direct cascade and Kolmogoroff scaling in the inertial regime.

Treating the field $u$ as the local slope of a growing interface, we analyzed in paper I, where a more complete bibliography can be found, the Burgers equation from the point of view of a soliton-carrying nonlinear damped evolution equation [28]. The equation describes the transient damped evolution of an initial slope configuration $u_{0}$ in terms of a gas of moving right hand viscositysmoothed solitons connected by ramp solutions and with superposed damped diffusive modes with a gap in their spectrum proportional to the soliton amplitudes. In a heuristic sense the transient morphology is thus characterized by two kinds of excitations: Nonlinear soliton modes and linear gapful diffusive modes. This picture is also borne out by the nonlinear Cole-Hopf transformation 2931

$$
w(x, t)=\exp \left[\frac{\lambda}{2 \nu} \int^{x} d x^{\prime} u\left(x^{\prime}, t\right)\right]
$$

\footnotetext{
${ }^{*}$ Permanent address
} 
relating the slope field $u$ to the diffusive field $w$ satisfying the linear diffusion equation

$$
\frac{\partial w}{\partial t}=\nu \nabla^{2} w
$$

with solution

$$
\begin{aligned}
w(x, t) & =\int d x^{\prime} G\left(x-x^{\prime}, t\right) w_{0}\left(x^{\prime}\right), \\
G(x, t) & =[4 \pi \nu t]^{-\frac{1}{2}} \exp \left[-x^{2} / 4 \nu t\right], \\
w_{0}(x) & =\exp \left[\frac{\lambda}{2 \nu} \int^{x} d x^{\prime} u_{0}\left(x^{\prime}\right)\right],
\end{aligned}
$$

allowing for a steepest descent analysis in the inviscid limit $\nu \rightarrow 0$ 23,24.

The emphasis on the nonlinear modes in paper I and the insight gained formed the natural starting point for our study of the noisy Burgers equation in paper II. This equation has the form [7, 8, 31, 33]

$$
\left(\frac{\partial}{\partial t}-\lambda u \nabla\right) u=\nu \nabla^{2} u+\nabla \eta
$$

where the Gaussian white noise driving the equation is spatially short range correlated according to

$$
\left\langle\eta(x, t) \eta\left(x^{\prime}, t^{\prime}\right)\right\rangle=\Delta \delta\left(x-x^{\prime}\right) \delta\left(t-t^{\prime}\right),
$$

characterized by the noise strength $\Delta$. Equation (11.7) has a much richer structure than the noiseless counterpart. The noise is here a singular perturbation in the sense that even a weak noise strength $\Delta$ eventually drives the morphology described by Eq. (1.7) into a stationary driven state; the characteristic time scale is set by $t \propto \ln (1 / \Delta)$ which diverges for $\Delta \rightarrow 0$. The singular nature of the noise is also reflected in the known stationary probability distribution for the slope field [31, 34 36]

$$
P_{\mathrm{st}}(u) \propto \exp \left[-\frac{\nu}{\Delta} \int d x u(x)^{2}\right] .
$$

Here $\Delta$ constitutes an essential singularity in the same manner as the temperature entering the usual Boltzmann factor.

Recasting the stochastic Langevin equation (1.7) in terms of a Martin-Siggia-Rose path integral [9 12, 14, 13 we proposed a principle of least action in the nonperturbative weak noise limit $\Delta \rightarrow 0$ and derived canonical saddle point or field equations (note that in paper II the noise field $\varphi$ was rotated, $\varphi \rightarrow-i \varphi)$

$$
\begin{aligned}
& \left(\frac{\partial}{\partial t}-\lambda u \nabla\right) u=\nu \nabla^{2} \varphi \\
& \left(\frac{\partial}{\partial t}-\lambda u \nabla\right) \varphi=\nu \nabla^{2} u
\end{aligned}
$$

coupling the slope field $u$ to a deterministic auxiliary noise field $\varphi$. The coupled field equations (1.10) and
(1.11) effectively replace the stochastic equation (1.7) and describe the morphology of a growing interface. In addition to the right hand soliton already present in the noiseless case as discussed in paper I, the field equations (1.10) and (1.11) also admit an equivalent left hand soliton. A growing interface can thus be viewed as a gas of connected right hand and left hand solitons with superposed diffusive modes. The weak noise approach also allows a dynamical description of the soliton and diffusive mode configurations and associates an action $S$, energy $E$, and momentum $\Pi$ with a particular interface morphology. The statistical weight of a configuration is given by $\exp [-S / \Delta]$ with the dynamical action $S$, providing the generalization of the Boltzmann factor $\exp [-E / T]$ for equilibrium processes to dynamical processes.

The Martin-Siggia-Rose path integral approach moreover permitted a simple interpretation of the scaling properties of a growing interface. The dynamical exponent $z=3 / 2$, entering in the dynamical scaling relation 31 33, 35, 39]

$$
\langle u(x, t) u(0,0)\rangle=|x|^{2 \zeta-2} F\left(|t| /|x|^{z}\right),
$$

is thus related to the gapless soliton dispersion law

$$
E \propto \lambda \Pi^{z},
$$

whereas the roughness exponent $\zeta=1 / 2$ follows from the spectral representation

$$
\langle u(x, t) u(0,0)\rangle=\int d K G(K) \exp [-i E t+i K x],
$$

assuming that the form factor $G(K) \sim$ const. in the scaling region $K \sim 0$. The dynamical scaling universality class is associated with the lowest gapless excitation, i.e., for $\lambda \neq 0$ the soliton mode. For $\lambda=0$ the EdwardsWilkinson (EW) universality class [31,36, 40 emerges with a gapless diffusive mode $\omega=\nu k^{2}$, corresponding to $z=2, \zeta=1 / 2$ being unaltered. Furthermore, we derived a heuristic expression for the scaling function $F$ in terms of the probability distribution for Lévy flights 41,42]; the scaling function has also been accessed by a mode coupling approach 43,44,.

Summarizing, the weak noise saddle point approach to the noisy Burgers equation advanced in paper II yields a many body description of the morphology of a growing interface in terms of two kinds of quasi-particles or elementary excitations: Nonlinear soliton modes corresponding to the faceted steplike growth of an interface with superposed linear diffusive modes. Furthermore, the scaling properties and the notion of universality classes follow as a byproduct from the dispersion law of the lowest gapless excitation. For details and references we refer the reader to the somewhat tutorial presentation in paper II.

Whereas a good understanding of the one dimensional case has been achieved both by renormalization group 
methods $7,8,31,33,37$ and $45,47,44$, by mapping to directed polymers [31, by mapping to spin chains 48 , 50 , from the lattice exclusion model [51 [53], and by the soliton approach in paper II, the general case in $d$ dimensions has proven much more difficult. In $d$ dimensions the noisy Burgers equation takes the form [7, 8$]$

$$
\left(\frac{\partial}{\partial t}-\lambda u_{m} \nabla_{m}\right) u_{n}=\nu \nabla^{2} u_{n}+\nabla_{n} \eta .
$$

Here the longitudinal vector field $u_{n}, n=1, . . d$, is associated with the height profile $h$ of a growing interface according to $\left(l_{n}\right.$ is a line element)

$$
\begin{aligned}
u_{n} & =\nabla_{n} h \\
h\left(x_{n}\right) & =\int^{x_{n}} d l_{n} u_{n} .
\end{aligned}
$$

Furthermore, $\nabla^{2}=\nabla_{n} \nabla_{n}$ and we assume summation over repeated Cartesian indices. The height field $h$ is thus the underlying potential for the force or slope field $u_{n}$. It follows from Eqs. (1.15) and (1.16) that $h$ satisfies the Kardar-Parisi-Zhang (KPZ) equation 31 33.

$$
\frac{\partial h}{\partial t}=\nu \nabla^{2} h+\frac{\lambda}{2} \nabla_{n} h \nabla_{n} h+F+\eta,
$$

for a growing interface in $d$ dimensions. Assuming $\langle\eta\rangle=$ 0 we have for completion here introduced the drift term $F=-(\lambda / 2)\left\langle\nabla_{n} h \nabla_{n} h\right\rangle$ in Eq. (1.17) in order to ensure that $\langle h\rangle$ decays in time in a co-moving frame. In Eqs. (1.15) and (1.17) $\nu$ is damping constant or viscosity characterizing the linear diffusive term, $\lambda$ is a coupling strength for the nonlinear mode coupling or growth term, and, finally, $\eta$ is a Gaussian white noise driving the equation and correlated according to

$$
\left\langle\eta\left(x_{n}, t\right) \eta\left(x_{n}^{\prime}, t^{\prime}\right)\right\rangle=\Delta \prod_{n} \delta\left(x_{n}-x_{n}^{\prime}\right) \delta\left(t-t^{\prime}\right)
$$

where $\Delta$ is the noise strength.

In higher dimension dynamic renormalization group calculations 31 33] yield a (lower) critical dimension at $d=2$ and a kinetic phase transition above $d=2$, separating a smooth phase characterized by the EW universality class yielding $\zeta=(2-d) / 2$ and $z=2$ and a rough phase characterized by a strong coupling fixed point. On the transition line renormalization group calculations and scaling arguments based on the mapping to directed polymers yield the exponents $\zeta=0$ and $z=2$ and suggest an upper critical dimension $d=4$ [54]. Most recently, an operator expansion method has been applied to the strong coupling phase yielding $(\zeta, z)=(2 / 5,8 / 5)$ in $d=2$ and $(\zeta, z)=(2 / 7,12 / 7)$ in $d=3$ 55,56.

In the present paper we focus on the stationary and time-dependent probability distributions for the height and slope fields described by the Burgers and KPZ equations (1.15) and (1.17), respectively. As discussed in paper II these distributions are basically given by the
Martin-Siggia-Rose path integral weighted by the effective action for the appropriate paths. In the weak noise limit only the paths governed by the saddle point equations contribute to the distributions and, as will be discussed here, we can actually circumvent the path integral formulation entirely by a more direct approach based on the Fokker-Planck equation. In the weak noise limit this equation takes the form of a Hamilton-Jacobi equation implying a symplectic structure and immediately lending itself to a canonical phase space formulation. We are thus able in a very direct manner to map the stochastic processes described by the KPZ-Burgers equations to a conserved dynamical system with orbits satisfying deterministic canonical Hamilton equations, identical to the saddle point equations in the path integral approach. The stochastic nature of the Langevin equations is reflected in the peculiar topology of the energy surfaces. It turns out that the stationary probability distribution is determined by an infinite-time orbit on the zero-energy manifold whereas the time-dependent distribution, approaching the stationary one at long times, corresponds to a finite-time orbit. Below we highlight some of our results.

(i) In the generic case of a general nonlinear Langevin equation for a set of stochastic variables driven by white noise, the weak noise limit of the associated FokkerPlanck equation takes the form of a Hamilton-Jacobi equation, which in turn implies a symplectic structure with a principle of least action, an action, an associated Hamiltonian, and Hamilton equations of motion. This formulation is equivalent to the saddle point discussion in the Martin-Siggia-Rose approach in paper II.

(ii) The ensuing canonical phase space formulation allows for a discussion of the time-dependent probability distributions, i.e., the weak noise solutions of the FokkerPlanck equation, in terms of phase space orbits on conserved energy surfaces, governed by Hamilton equations of motion. The action associated with an orbit plays the role of a weight function in much the same way as the Hamiltonian entering the Boltzmann factor in the description of thermodynamic equilibrium. In the kinetic nonequilibrium problem defined by the Langevin equation the dynamic action yields the probability distributions.

(iii) In the canonical phase space formulation the underlying stochastic nature of the Langevin equation and the relaxational character of the solutions of the FokkerPlanck equation are reflected in the topological structure of the energy surfaces. A structure which differs markedly from the energy surface topology for ordinary dynamical problems In particular, the zero-energy manifold which determines the stationary state, i.e., the stationary probability distribution, has a two-fold submanifold structure, including a hyperbolic stationary point, which in the simple case of a single stochastic variable corresponds to the unstable maximum of an inverted potential. Moreover, the waiting time for the orbits passing close to the stationary point accounts for the Marko- 
vian behavior of the probability distributions. Finally, the long-time orbit close to the zero-energy manifold determines via the action the time-dependent probability distribution.

(iv) In the case of a few degrees of freedom the canonical phase space approach yields the established results following from an analysis of the Fokker-Planck equations. On the other hand, in the case of many degrees of freedom, i.e., the field theoretical case, the FokkerPlanck equation becomes an unwieldy multi- dimensional differential-integro equation, and the canonical phase space approach, replacing the Fokker-Planck equation (in the weak noise limit) with coupled canonical field equations yields, in addition to providing an alternative point of view of the stochastic processes in terms of dynamical system theory, a methodological advantage; particularly in the case where we can determine the zero-energy manifold explicitly.

(v) In the field theoretical cases of the noisy Burgers or KPZ equations in one dimension, we can for special reasons identify the zero-energy manifold and determine: (1) the stationary distribution, (2) the long-time diffusive mode contribution to the time-dependent skew distribution and (3) a heuristic expression for the short-time (transient) soliton mode contribution. In the interesting case of higher dimensions we are only able to make some general statements.

The paper is organized in the following manner. In Sec II we consider the generic case of a nonlinear Langevin equation for many stochastic variables driven by white noise. We analyze the associated Fokker-Planck equation in the weak noise limit and set up the canonical phase space formulation. In Sec. III we consider as an example the stochastic overdamped motion in a harmonic potential. In Sec. IV we apply the formulation to the Burgers and KPZ equations and derive expressions for the distributions. Finally, in Sec $\mathrm{V}$ we present a discussion and a conclusion.

\section{THE CANONICAL PHASE SPACE APPROACH}

Path integral formulations of the Fokker-Planck equation in the field theoretical case and aspects of the canonical structure have been discussed in the literature, see [57] for further referencing, we believe, however, that the present emphasis on the canonical phase space formulation as a practical tool is new. For this purpose we here set up the general canonical phase space formalism. Adhering to the notation in 57] we consider a general Langevin equation with additive noise [58,59,

$$
\frac{d q_{n}}{d t}=-\frac{1}{2} F_{n}\left(q_{m}\right)+\eta_{n} .
$$

Here $q_{n}, n=1, . . N$, is a set of time-dependent stochastic variables. The index $n$ is discrete but is readily generalized later to the field theoretical case of infinitely many degrees of freedom where $n$ typically includes the spatial variables. The forces $F_{n}\left(q_{m}\right)$ are general functions of $q_{n}$. In the linear case of coupled (overdamped) oscillators, $F_{n}=2 \Omega_{n m} q_{m}(t)$, where $\Omega_{n m}$ is a damping matrix. Finally, the equation is driven stochastically by a white noise term $\eta_{n}$ with a Gaussian distribution and correlated according to

$$
\left\langle\eta_{n}(t) \eta_{m}\left(t^{\prime}\right)\right\rangle=\Delta K_{n m} \delta\left(t-t^{\prime}\right)
$$

Here $K_{n m}$ is a constant, symmetric, positive-definite noise matrix of $O(1)$ and the correlations are characterized by the noise strength $\Delta$.

Introducing the notation $\nabla_{n}=\partial / \partial q_{n}$ the FokkerPlanck equation for the (conditional) probability distribution $P\left(q_{n}, t, q_{n}^{\prime}\right)$ associated with Eq. 2.1) has the form 5759

$$
\frac{\partial P}{\partial t}=\frac{1}{2} \nabla_{n}\left[F_{n} P+\Delta K_{n m} \nabla_{m} P\right],
$$

including a drift term $\nabla_{n}\left(F_{n} P\right)$, arising from the deterministic force $F_{n}$, and a diffusion term $\Delta K_{n m} \nabla_{n} \nabla_{m} P$, originating from the noise $\eta_{n}$.

In the equilibrium case, choosing $K_{n m}=\delta_{n m}$ and setting $F_{n}=\nabla_{n} \Phi$, corresponding to an effective fluctuationdissipation theorem and an underlying thermodynamic free energy $\Phi$, Eq. (2.3) admits the stationary solution $P_{s t} \propto \exp [-\Phi / \Delta]$ with $\Delta$ entering as a temperature and mathematically as a singular parameter, i.e., the Boltzmann distribution. Using this as a guiding principle we search in the general nonequilibrium case for solutions to Eq. 2.3) of the form

$$
P \propto \exp \left[-\frac{1}{\Delta} S\right]
$$

where the weight function $S$ replaces the free energy $\Phi$ in the equilibrium case. By insertion and keeping only terms to leading order in $\Delta$, it is easy to show that $S$ satisfies an equation of the Hamilton-Jacobi form 60 62],

$$
\frac{\partial S}{\partial t}+H\left(q_{n}, \nabla_{n} S\right)=0,
$$

where, introducing the canonical momentum and energy

$$
\begin{gathered}
p_{n}=\nabla_{n} S, \\
E=H,
\end{gathered}
$$

the conserved energy or Hamiltonian is given by

$$
H=\frac{1}{2} K_{n m} p_{n} p_{m}-\frac{1}{2} F_{n} p_{n} .
$$

From the symplectic structure and dynamical system theory the canonical phase space structure follows immediately. The action $S$ has the form 60

$$
S=\int d t\left(p_{n} \frac{d q_{n}}{d t}-H\right)
$$


and from the ensuing principle of least action we derive the Hamiltonian equations of motion, $d q_{n} / d t=\partial H / \partial p_{n}$ and $d p_{n} / d t=-\partial H / \partial q_{n}$,

$$
\begin{aligned}
\frac{d q_{n}}{d t} & =K_{n m} p_{m}-\frac{1}{2} F_{n}, \\
\frac{d p_{n}}{d t} & =\frac{1}{2} p_{m} \nabla_{n} F_{m},
\end{aligned}
$$

for the orbits in $p_{n} q_{n}$ phase space.

The above formulation allows a simple interpretation of the solution of the Fokker-Planck equation (2.3) in the weak noise limit $\Delta \rightarrow 0$ in terms of orbits in a canonical phase space. In order to determine the transition probability $P\left(q_{n}, T, q_{n}^{\prime}\right)$ for a configuration $q_{n}^{\prime}$ at $t=0$ to a configuration $q_{n}$ at $t=T$, we simply solve the Hamilton equations (2.10) and (2.11) for an orbit from $q_{n}^{\prime}$ to $q_{n}$ traversed in time $T$ and, subsequently, evaluate the action according to Eq. (2.9), yielding the weight function in Eq. (2.4), i.e.,

$$
P\left(q_{n}, T, q_{n}^{\prime}\right) \propto \exp \left[-\frac{1}{\Delta} \int_{0, q_{n}^{\prime}}^{T, q_{n}} d t\left(p_{n} \frac{d q_{n}}{d t}-H\right)\right] .
$$

We notice that the relevant orbit is determined by the initial and final values $q_{n}^{\prime}$ and $q_{n}$ and the elapsed time $T$. The canonically conjugate momentum $p_{n}$ is a slaved variable determined by Eq. (2.11) and parametrically coupled to Eq. (2.10). Also, unlike the case in ordinary mechanics, the energy $E=H$ in Eq. (2.8) is not the central quantity in the present interpretation. The traversal time $T$ is the important variable and the energy manifold $E(T)$ on which the orbit from $q_{n}^{\prime}$ to $q_{n}$ lies is a function of $T$.

The stochastic nature of the Langevin equation (2.1) and the properties of the weak noise solution of the Fokker-Planck equation (2.3) are reflected in the topological submanifold structure of the energy surfaces in $p_{n} q_{n}$ phase space. Unlike an ordinary mechanical problem $H$ is not bounded from below and does not separate in a kinetic energy and a potential energy only depending on $q_{n}$. In Eq. (2.8) the potential $-(1 / 2) F_{n} p_{n}$ is momentum (velocity) dependent and gives rise to unbounded motion. Assuming for simplicity that $F_{n} \rightarrow 0$ for $q_{n} \rightarrow 0$ the energy surfaces have the submanifold structure depicted in Fig 1.

The origo in phase space constitutes a hyperbolic stationary point, that is a saddle point determined by the zero-energy submanifold $p_{n}=0$ and the zero-energy submanifold defined by $K_{n m} p_{m}-F_{n}$ being orthogonal to $p_{n}$, i.e., $K_{n m} p_{m}-F_{n} \perp p_{n}$.

Assuming $F_{n}\left(q_{m}\right) \sim 2 \Omega_{n m} q_{m}$ for small $q_{n}$ the Hamiltonian (2.8) is quadratic in $p_{n}$ and $q_{n}$ and a stability analysis can easily be carried out. In accordance with the present physical interpretation we assume that the stability or damping matrix $\Omega_{n m}$ implies an unstable $p_{n}=0$ submanifold and a stable submanifold $K_{m n} p_{m}-F_{n} \perp p_{n}$.
The orbits in phase space close to the zero-energy manifold are thus those depicted in Fig. 1. In the harmonic oscillator picture which applies close to the stationary point this behavior corresponds to the motion in an inverted parabolic potential as discussed in more detail in Sec. III.

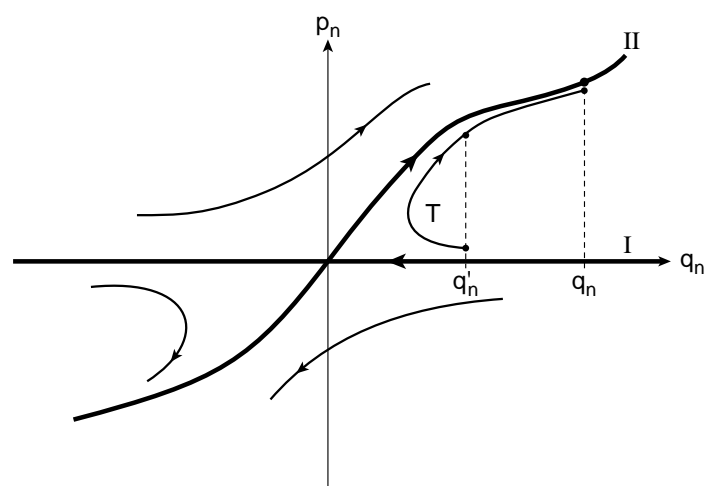

FIG. 1. Canonical phase space in the general case. The solid curves indicate the zero-energy transient submanifold (I) and stationary submanifold (II). The stationary saddle point is at the origin. The finite time $(T)$ orbit from $q_{n}^{\prime}$ to $q_{n}$ migrates to the zero-energy submanifold for $T \rightarrow \infty$.

The stationary state is given by orbits on the zeroenergy manifold whose structure thus determines the nature of the stochastic problem. Assuming that $E(T) \propto$ $\exp [-$ const. $T]$ for $T \rightarrow \infty$ the stationary state

$$
P_{\mathrm{st}}\left(q_{n}\right)=\lim _{T \rightarrow \infty} P\left(q_{n}, T, q_{n}^{\prime}\right),
$$

is obtained from Eq. (2.12), i.e.,

$$
P_{\mathrm{st}}\left(q_{n}\right) \propto \exp \left[-\frac{1}{\Delta} \int_{0}^{\infty} d t p_{n} \frac{d q_{n}}{d t}\right] .
$$

We note that for $T \rightarrow \infty$ the orbit from $q_{n}^{\prime}$ to $q_{n}$ converges to the zero-energy manifold, i.e., $P_{\text {st }}$ is determined by the infinite-time orbits on the zero-energy manifold.

The basic structure of phase space, depicted in Fig. 1, allows for a simple dynamical discussion in terms of dynamical system theory [18,62, 63. of the approach to the stationary state of a damped noise-driven stochastic system. We first consider an orbit on an $E=0$ surface from $q_{n}^{\prime}$ to $q_{n}$ in time $T$. The energy surface $E(T)$ depends on $T$ and in the limit $T \rightarrow \infty, E \rightarrow 0$ in order to attain the stationary state. For $E \rightarrow 0$ the initial part of the orbit moves close to the $p_{n}=0$ submanifold and from Eq. (2.10) is determined by

$$
\frac{d q_{n}}{d t}=-\frac{1}{2} F_{n}
$$

i.e., the deterministic noiseless version of the Langevin equation (2.1). In the absence of noise the motion is transient and damped. Near the transient submanifold 
$p_{n}=0$ the corresponding action $S \sim 0$ and the probability $P=$ const., corresponding to a deterministic behavior. The orbit slows down near the stationary point in phase space before it picks up again and moves close to the other stationary submanifold $K_{n m} p_{m}-F_{n} \perp p_{n}$. This part of the orbit carries a finite action $S$, i.e., $P$ depends on $q_{n}$, terminates in $q_{n}$ at time $T$, and corresponds for $T \rightarrow \infty$ to the stationary state. The Markovian behavior, i.e., the loss of memory or the independence of the initial configuration $q_{n}^{\prime}$, is thus associated with the long (infinite) waiting time near (at) the stationary point.

Whereas the transient submanifold $p_{n}=0$, yielding $E=0$, is consistent with the Hamiltonian equations 2.10) and (2.11) and gives rise to the deterministic equation (2.15), the other possibility of imposing a zero-energy submanifold by setting $K_{n m} p_{m}=F_{n}$ will in general violate Eqs. (2.10) and (2.11). Assuming for simplicity $K_{n m}=\delta_{n m}$ (note that the symmetric noise matrix $K_{n m}$ can always be diagonalized by a suitable choice of $p_{n}$ ) we obtain, inserting $p_{n}=F_{n}$ in Eqs. (2.10) and (2.11), that the relationship $F_{m}\left(\nabla_{m} F_{n}-\nabla_{n} F_{m}\right)=0$ must hold. In the special case where $F_{n}=\nabla_{n} \Phi$, corresponding to an effective fluctuation-dissipation theorem and an underlying free energy $\Phi$, the above constraint is trivially satisfied and we obtain the time-reversed equation of motion $d q_{n} / d t=(1 / 2) F_{n}$ governing the orbit on the $p_{n}=F_{n}$ stationary submanifold. It is interesting to notice that the damped motion on the transient $p_{n}=0$ submanifold is precisely equal to the time-reversed growing motion on the stationary $p_{n}=F_{n}$ submanifold. Finally, it is an easy task to determine the stationary distribution

$$
P_{\text {st }} \propto \exp \left[-\frac{1}{\Delta} \Phi\left(q_{n}\right)\right]
$$

by insertion of $p_{n}=\nabla_{n} \Phi$ in Eq. 2.14 and integrating over time., in agreement with the solution of the Fokker-Planck equation (2.3) in the stationary case set$\operatorname{ting} K_{n m}=\delta_{n m}$ and $F_{n}=\nabla_{n} \Phi$.

We are led to the conjecture that for systems in thermal equilibrium where the force $F_{n}$ is derived from a thermodynamic free energy $\Phi, F_{n}=\nabla_{n} \Phi$, the infinitetime orbits on the stationary zero-energy submanifold actually converge to the submanifold $p_{n}=F_{n}$, yielding the stationary distribution (2.16). In the general case of a driven stochastic system with a force $F_{n}$ not derivable from a free energy the only constraint is given by $K_{n m} p_{m}-F_{n} \perp p_{n}$ and we have to solve the Hamilton equations 2.10) and (2.11) in order to determine the phase space orbits.

We finally wish to comment on the connection between the present canonical phase space approach and the formulation in terms of a Martin-Siggia-Rose (MSR) path integral presented in paper II. For simplicity we consider only the case of a single stochastic degree of freedom $q$ and set the noise matrix $K_{n m}=1$.

All the relevant properties are extracted from the generator 57

$$
Z(\mu)=\left\langle\exp \left[i \int d t \mu(t) q(t)\right]\right\rangle
$$

where $\langle\cdots\rangle$ denotes an average with respect to the Gaussian noise distribution

$$
P(\eta) \propto \exp \left[-\frac{1}{2 \Delta} \int d t \eta(t)^{2}\right] .
$$

The Langevin equation (2.1) for one degree of freedom is enforced by the delta function constraint $\int \prod_{t} d t J \delta(d q / d t+(1 / 2) F-\eta)=1$, where the Jacobian $J=\exp \left[(1 / 4) \int d t d F / d q\right]$ [57]. Exponentiating the constraint and in the process introducing an additional noise variable $p$, averaging over the noise $\eta$ according to Eq. (2.18), and scaling $p, p \rightarrow p / \Delta$, we obtain the expression

$$
Z(\mu) \propto \int \prod_{t} d p d q \exp \left[\frac{i}{\Delta} S_{\mathrm{MSR}}\right] \exp \left[i \int d t \mu(t) q(t)\right]
$$

where the action has the Feynman form 64, 65

$$
S_{\mathrm{MSR}}=\int d t\left(p \frac{d q}{d t}-H_{\mathrm{MSR}}\right),
$$

with complex Hamiltonian

$$
H_{\mathrm{MSR}}=-\frac{1}{2} p F-\frac{i}{2} p^{2}+i \frac{\Delta}{4} \frac{d F}{d q} .
$$

For the probability distribution $P\left(q, t, q^{\prime}\right)$ we find in particular

$$
P\left(q, t, q^{\prime}\right) \propto \int_{q^{\prime}}^{q} \prod_{t} d p d q \exp \left[\frac{i}{\Delta} S_{\mathrm{MSR}}\right]
$$

where $S_{\mathrm{MSR}}=\int_{0}^{t} d t\left(p d q / d t-H_{\mathrm{MSR}}\right)$ and the path integral samples all orbits from $q^{\prime}=q(0)$ to $q=q(t)$ weighted with $S_{\mathrm{MRS}}$; note that the noise field ranges freely.

Reconstructing the underlying quantum mechan$i c s$, yielding the path integral (2.22), $P\left(q, t, q^{\prime}\right)$ can be regarded as a matrix element of the evolution operator $\exp \left[-i \hat{H}_{\mathrm{MSR}} t\right]$ in a q-basis, $P\left(q, t, q^{\prime}\right) \propto$ $\left\langle q\left|\exp \left(-i \hat{H}_{\mathrm{MSR}} t\right)\right| q^{\prime}\right\rangle$, where $\hat{H}_{\mathrm{MSR}}$ is the quantum version of $H_{\mathrm{MSR}}$. It follows that $P\left(q, t, q^{\prime}\right)$ then satisfies the Schrödinger equation

$$
i \Delta \frac{\partial P}{\partial t}=\hat{H}_{\mathrm{MSR}} P
$$

with $\Delta$ playing the role of an effective Planck constant. The noise variable $p$ becomes the momentum operator $\hat{p}=-i \Delta d / d q$ and we obtain, inserting $p \rightarrow \hat{p}$ in Eq. (2.21),

$$
\hat{H}_{\mathrm{MSR}}=i \frac{1}{2}\left[\Delta^{2} \frac{d^{2}}{d q^{2}}+i(\hat{p} F)_{\mathrm{order}}+\frac{\Delta}{2} \frac{d F}{d q}\right]
$$


where since $[\hat{p}, q]=-i \Delta$ and $F$ depends on $q$ we still have to specify the ordering in $(\hat{p} F)_{\text {order }}$.

Comparing Eqs. (2.23) and (2.24) with the FokkerPlanck equation (2.3) in the present case,

$$
\frac{\partial P}{\partial t}=\frac{1}{2}\left[\Delta \frac{d^{2}}{d q^{2}}+F \frac{d}{d q}+\frac{d F}{d q}\right] P,
$$

we find agreement provided we choose the symmetric ordering $(\hat{p} F)_{\text {order }}=\frac{1}{2}(\hat{p} F+F \hat{p})$. Alternatively, we are free to choose a normal ordering $(\hat{p} F)_{\text {order }}=\hat{p} F$ and neglect the Jacobian contribution $(\Delta / 2) d F / d q$ in Eq. (2.24). The Fokker-Planck equation then becomes the underlying Schrödinger equation for the path integral with a complex non Hermitian Hamiltonian,

$$
\hat{H}_{\mathrm{FP}}=i \frac{1}{2}\left[\Delta^{2} \frac{d^{2}}{d q^{2}}+\Delta \frac{d}{d q} F\right] .
$$

The non Hermitian form of $\hat{H}_{\mathrm{FP}}$ with the $\hat{p}$ operator on the left ensures that Eq. (2.25) has the form of a conservation law ensuring the conservation of probability.

In the limiting case $F=2 \omega q$ the Hamiltonian $H_{\mathrm{MSR}}$ describes a harmonic oscillator and it is easy to see that the Jacobian contribution $i(\Delta / 4) d F / d q=i(\Delta / 2) \omega$ in

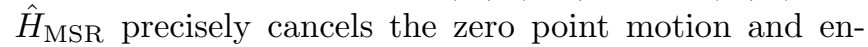
sures a stationary state for $t \rightarrow \infty$.

Finally, in the classical weak noise limit for $\Delta \rightarrow 0$, the path integral (2.22) is dominated by the classical or stationary orbits following from the principle of least action $\delta S_{\mathrm{MSR}}=0$ and determined as solutions of the Hamilton equations of motion: $d q / d t=\partial H_{\mathrm{MSR}} / \partial p$ and $d p / d t=-\partial H_{\mathrm{MSR}} / \partial q$; the distribution being given by $P \propto \exp \left[(i / \Delta) S_{\mathrm{MSR}}\right]$. This procedure is, however, entirely equivalent to performing the limit $\Delta \rightarrow 0$ directly in the Fokker-Planck equation, yielding the HamiltonJacobi equation (2.5) and the present canonical phase space approach.

\section{THE HARMONIC OSCILLATOR - AN EXAMPLE}

In order to illustrate the phase space method we here apply it to the simple case of an overdamped harmonic oscillator described by the Langevin equation and noise correlations

$$
\begin{aligned}
& \frac{d q}{d t}=-\omega q+\eta, \\
& \left\langle\eta(t) \eta\left(t^{\prime}\right)\right\rangle=\Delta \delta\left(t-t^{\prime}\right),
\end{aligned}
$$

with associated Fokker-Planck equation

$$
\frac{\partial P}{\partial t}=\frac{1}{2} \frac{\partial}{\partial q}\left[\Delta \frac{\partial P}{\partial q}+2 \omega P\right] .
$$

This system is well-known and easily analyzed [57,58]. The time-dependent probability distribution $P\left(q, T, q^{\prime}\right)$, the solution of Eq. (3.3), is given by

$$
P\left(q, T, q^{\prime}\right) \propto \exp \left[-\frac{\omega}{\Delta} \frac{\left[q-q^{\prime} \exp (-\omega T)\right]^{2}}{1-\exp (-2 \omega T)}\right],
$$

approaching the stationary distribution

$$
P_{\mathrm{st}}(q) \propto \exp \left[-\frac{1}{\Delta} \omega q^{2}\right],
$$

in the limit $T \rightarrow \infty$.

We now proceed to derive these results within the canonical phase space formulation. Since $F=2 \omega q$ and $K=1$ for one degree of freedom, we obtain from Eqs. (2.8) - 2.11) the Hamiltonian

$$
H=\frac{1}{2} p^{2}-\omega q p
$$

the action

$$
S=\int d t\left(p \frac{d q}{d t}-H\right)
$$

and the Hamilton equations of motion

$$
\begin{aligned}
& \frac{d q}{d t}=p-\omega q \\
& \frac{d p}{d t}=\omega p .
\end{aligned}
$$

The phase space is depicted in Fig. 2 and corresponds to the vicinity of the stationary point in Fig. 1 for one degree of freedom.

For a single degree of freedom we can explicitly determine both zero-energy submanifolds: $p=0$ and $p=2 \omega q$, and determine the orbits. On the transient $p=0$ submanifold $d q / d t=-\omega q$, i.e., the deterministic equation of motion for $\eta=0$, with a damped solution $q=q_{0} \exp [-\omega t], q_{0}=q(0)$, corresponding to the damped orbit approaching the stationary saddle point at $(p, q)=(0,0)$. The action associated with this orbit is $S=0$, i.e., $P=$ const., characterizing the deterministic motion. On the stationary submanifold $p=2 \omega q$ the Hamiltonian equations coincide, $d q / d t=2 \omega q$, and we obtain a growing solution $q=q_{0} \exp [\omega t], q_{0}=q(0)$, associated with the orbit emerging from the stationary point. As discussed in Sec. II we note that the stationary orbit is the time-reversed mirror of the transient orbit.

The complete solution of Eqs. (3.8) and (3.9) is also easily obtained. For an orbit from $q^{\prime}$ to $q^{\prime \prime}$ in time $T$ and, noting that $p$ is a slaved variable, we obtain

$$
\begin{aligned}
& q(t)=\frac{q^{\prime \prime} \sinh \omega t+q^{\prime} \sinh \omega(T-t)}{\sinh \omega T}, \\
& p(t)=\omega \frac{q^{\prime \prime} e^{\omega t}-q^{\prime} e^{-\omega(t-T)}}{\sinh \omega T} .
\end{aligned}
$$




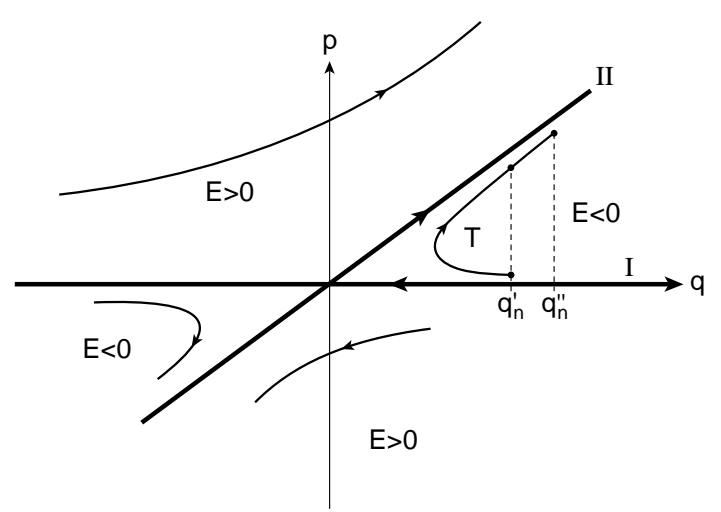

FIG. 2. Canonical phase space plot in the case of an overdamped oscillator. The solid curves indicate the zero-energy transient submanifold (I) and stationary submanifold (II). The stationary saddle point is at the origin. The finite time $(T)$ orbit from $q_{n}^{\prime}$ to $q_{n}$ migrates to the zero-energy submanifold for $T \rightarrow \infty$. We have also indicted the sign of the energy $E$ in the four domains.

For large $T$ the noise variable $p$ is initially close to zero, corresponding to the transient deterministic regime; for $t$ close to $T p$ eventually leaves zero and approaches the limiting value $2 \omega q^{\prime \prime}$, corresponding to the stationary regime. Likewise, we note that $q \sim 0$ for $T \rightarrow \infty$ for most $t$. The behavior of $q$ and $p$ is depicted in Fig. 3 .
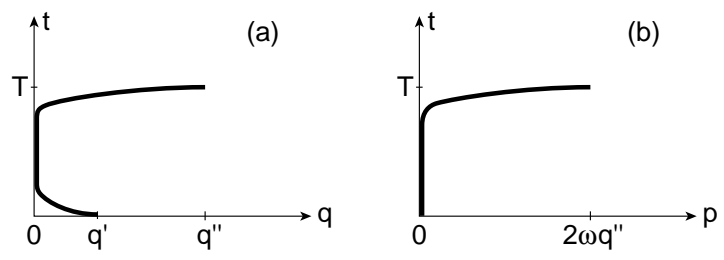

FIG. 3. Plot of $q$ and $p$ as functions of $t$ in the case of an overdamped oscillator. In a) we depict the dependence of $q$; for large $T$ the coordinate $q$ stays close to the stationary saddle point. In b) we show the dependence of $p$; for large $T$ the momentum $p$ is initially close to the transient submanifold $p=0$ but eventually moves on to the stationary submanifold $p=2 \omega q$.

The orbit from $q^{\prime}$ to $q^{\prime \prime}$ traversed in time $T$ lies on the energy surface given by

$$
E=\frac{\omega^{2}}{2} \frac{q^{\prime \prime 2}+q^{\prime 2}-2 q^{\prime} q^{\prime \prime} \cosh \omega T}{\sinh ^{2} \omega T} .
$$

For fixed $q^{\prime}$ and $q^{\prime \prime}$ the energy $E$ is a function of $T$, $E=E(T)$. In the limit $T \rightarrow \infty, E \rightarrow 0$ and the orbit converges to the zero-energy manifold as indicated in Fig. 2. The actual $T=\infty$ orbit passing through the stationary point is then defined by the limiting orbit for $T \rightarrow \infty$.

Moreover, from the Hamilton equations (3.8) and (3.9) we readily deduce the second order Newton equation of motion

$$
\frac{d^{2} q}{d t^{2}}=\omega^{2} q
$$

describing the orbit in an inverted harmonic potential $-(1 / 2) \omega^{2} q^{2}$, and allowing for a simple discussion of the motion in $p q$ phase space.

The finite energy orbits fall in two categories depending on the sign of $E$. For $E>0$ the orbits pass through the unstable maximum of the inverted potential with finite momentum; for $E<0$ the unbounded orbits are confined by the potential to either positive or negative values of $q$. The limiting case $E=0$ corresponds to an orbit approaching the maximum, the hyperbolic stationary point, with zero momentum. This point represents an unstable equilibrium where the particle spends an infinite amount of time, corresponding to the establishment of Markovian behavior, i.e., the loss of memory and independence of the initial configuration $q^{\prime \prime}$. The motion is depicted in Fig. 4.

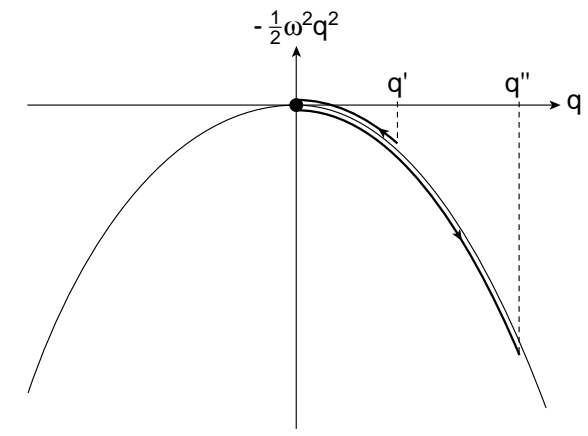

FIG. 4. In the case of the overdamped oscillator the orbits in $p q$ phase space depicted in Fig. 2 corresponds in $q$ space to the motion in an inverted parabolic potential. The unstable maximum corresponds to the stationary saddle point.

In terms of the explicit solution (3.10) and (3.11) we finally derive the action associated with the orbit,

$$
S=\omega \frac{\left(q^{\prime \prime}-q^{\prime} e^{-\omega T}\right)^{2}}{1-e^{-2 \omega T}},
$$

and recover from $P \propto \exp [-S / \Delta]$ the time-dependent and in the limit $T \rightarrow \infty$, stationary distributions (3.4) and $(3.5)$, respectively.

The Hamiltonian (3.6) and the equations of motion (3.8) and (3.9), yielding the canonical phase space representation of the Langevin equation for a noise-driven overdamped harmonic oscillator, have the same structure as the dynamical description of an ordinary harmonic oscillator. Shifting the momentum, $p \rightarrow p+\omega q$, 
$H \rightarrow(1 / 2) p^{2}-(1 / 2) \omega q^{2}$, describing the motion in an inverted harmonic potential as discussed above. The equations of motion now take the form $d q / d t=p$ and $d p / d t=\omega q$ with solutions given as linear combinations of a growing and a damped solution as in Eqs. (3.10) and (3.11). However, performing a Wick rotation $t \rightarrow i t=\tau$ in combination with the transformation to a complex momentum $p \rightarrow-i p$, note that $p$ is basically a dummy variable representing the noise, we obtain $H \rightarrow-H_{\mathrm{osc}}$, where $H_{\mathrm{osc}}$ is the oscillator Hamiltonian $H_{\mathrm{osc}}=\frac{1}{2} p^{2}+\frac{1}{2} \omega^{2} q^{2}$, yielding the equations of motion $d q / d \tau=p$, and $d p / d \tau=-\omega q$ with bounded periodic motion in imaginary time $\tau$. The energy $E=H_{\text {osc }}$ is positive and the finite energy orbits in $p q$ phase space move on concentric ellipses. The zero-energy manifold corresponds to the origin $(p, q)=(0,0)$ in phase space. In fact, subject to the Wick rotation $t \rightarrow i t$ and a rotation of $p, p \rightarrow-i p$, this phase space structure is mapped to the phase space structure in Fig. 2 with a hyperbolic stationary point and unbounded orbits. As pointed out in Sec. II it is precisely in the energy surface topology that the stochastic problem differs from an ordinary dynamical problem; here exemplified in the context of the oscillator. Correspondingly, the action (3.7) for an orbit from $q^{\prime}$ to $q^{\prime \prime}$ in time $i T$ transforms to $-i S_{\text {osc }}+(\omega / 2)\left(q^{\prime \prime 2}-q^{\prime 2}\right)$ where $S_{\text {osc }}$ is the action for a harmonic oscillator 66. 665

$$
S_{\mathrm{osc}}=\frac{\omega}{2 \sin \omega T}\left[\left(q^{\prime \prime 2}+q^{\prime 2}\right) \cos \omega T-2 q^{\prime} q^{\prime \prime}\right],
$$

yielding $P\left(q^{\prime \prime}, i T, q^{\prime}\right)$ in accordance with with Eq. (3.4).

Finally, we present a simple calculation of the leading correction at long times to the stationary distribution (3.5) which will prove useful in our discussion of the Burgers-KPZ equations in the next section. For $T \rightarrow \infty$ the orbit in phase space is close to the stationary zero-energy submanifold $p=2 \omega q$. Replacing the orbit from $q^{\prime}$ to $q^{\prime \prime}$ at long times $T$ with an orbit on the stationary manifold we obtain a constraint which allows us to simply evaluate the correction to $P_{\mathrm{st}}$. Consequently, inserting the zero-energy constraint $p=2 \omega q$ in the canonical equation (3.8) yields $d q / d t=\omega q$ with solution $q^{\prime \prime}=\exp (\omega T) q^{\prime}$ for $q^{\prime}$ and $q^{\prime \prime}$ on the $p=2 \omega q$ manifold. Inserting this solution in the action (3.7) we obtain

$$
S=\omega\left(q^{\prime \prime 2}-q^{\prime 2}\right)=\omega q^{\prime 2}(1-\exp (-2 \omega T)),
$$

in accordance with an expansion of the exact result (3.14) to leading order in $\exp (-\omega T)$.

\section{CANONICAL FORMULATION OF THE BURGERS - KPZ EQUATIONS}

In this section we apply the canonical phase space method developed in Sec. II to the Burgers and KPZ equations (1.15) and (1.17).

\section{A. The general case}

First applying the canonical formulation to the Burgers equation (1.15) the index $n$ in Sec. II now comprises both the continuous spatial coordinate $x_{n}, n=1, . . d$ and the vector index of the slope field $u_{n}, n=1, . . d$, i.e., $n \rightarrow$ $x_{n}, n$. Furthermore, we choose the noise matrix $K_{n m}$ and the forces $F_{n}$ according to the prescription, $K_{n m} \rightarrow$ $\nabla^{2} \prod_{n} \delta\left(x_{n}-x_{n}^{\prime}\right)$ and $F_{n} \rightarrow-2\left(\nu \nabla^{2} u_{n}+\lambda u_{m} \nabla_{m} u_{n}\right)$. With the identification $q_{n} \rightarrow u_{n}\left(x_{m}\right)$ and $p_{n} \rightarrow p_{n}\left(x_{m}\right)$ we thus obtain the Burgers action and Hamiltonian density

$$
\begin{aligned}
S_{\mathrm{B}} & =\int_{0}^{T} d^{d} x d t\left(p_{n} \frac{\partial u_{n}}{\partial t}-\mathcal{H}_{\mathrm{B}}\right), \\
\mathcal{H}_{\mathrm{B}} & =p_{n}\left(\nu \nabla^{2} u_{n}+\lambda u_{m} \nabla_{m} u_{n}-\frac{1}{2} \nabla_{n} \nabla_{m} p_{m}\right),
\end{aligned}
$$

and the ensuing Hamilton equations of motion

$$
\begin{aligned}
\left(\frac{\partial}{\partial t}-\lambda u_{m} \nabla_{m}\right) u_{n}= & \nu \nabla^{2} u_{n}-\nabla_{n} \nabla_{m} p_{m} \\
\left(\frac{\partial}{\partial t}-\lambda u_{m} \nabla_{m}\right) p_{n}= & -\nu \nabla^{2} p_{n} \\
& +\lambda\left(p_{n} \nabla_{m} u_{m}-p_{m} \nabla_{n} u_{m}\right) .
\end{aligned}
$$

The time-dependent probability distribution is then in the weak noise limit given by

$$
P\left(u_{n}, T, u_{n}^{\prime}\right) \propto \exp \left[-\frac{1}{\Delta} S_{\mathrm{B}}\left(u_{n}, T, u_{n}^{\prime}\right)\right] .
$$

The Hamilton equations (4.3) and (4.4) determining the orbits in $p_{n} u_{n}$ phase space thus replace the noisy Burgers equation (1.15) in the weak noise limit and the distribution (4.5), evaluated for an appropriate orbit from $u_{n}^{\prime}$ to $u_{n}$ traversed in time $T$, constitutes a weak noise solution of the Fokker-Planck equation associated with the Burgers equation,

$$
\begin{aligned}
& \frac{\partial P\left(u_{n}, t\right)}{\partial t}= \\
& -\int d^{d} x \frac{\delta}{\delta u_{n}}\left[\left(\nu \nabla^{2} u_{n}+\lambda u_{m} \nabla_{m} u_{n}\right) P\left(u_{n}, t\right)\right] \\
& +\frac{\Delta}{2} \int d^{d} x d^{d} x^{\prime} \frac{\delta^{2}}{\delta u_{n} \delta u_{n}^{\prime}}\left[\nabla^{2} \prod_{n} \delta\left(x_{n}-x_{x}^{\prime}\right) P\left(u_{n}, t\right)\right] .
\end{aligned}
$$

The time-dependent and stationary distributions are determined by the orbits near and on the zero-energy manifold. From the general discussion in Sec. II it follows that the zero-energy manifold has a submanifold structure with a transient $p_{n}=0$ submanifold, a hyperbolic stationary point at $\left(u_{n}, p_{n}\right)=(0,0)$, and a stationary submanifold defined by $\nu \nabla^{2} u_{n}+\lambda u_{m} \nabla_{m} u_{n}-(1 / 2) \nabla_{n} \nabla_{m} p_{m}$ 
orthogonal to $p_{n}$; here treating the integration over $x$ in $\int d^{d} x \mathcal{H}_{B}$ as an inner product. On the transient submanifold $p_{n}=0 \mathrm{Eq}$. (4.4) is trivially satisfied and the orbits are governed by the noiseless Burgers equation

$$
\left(\frac{\partial}{\partial t}-\lambda u_{m} \nabla_{m}\right) u_{n}=\nu \nabla^{2} u_{n}
$$

which is analyzed by means of the Cole-Hopf transformation (1.2) $u_{n}=\nabla_{n} h, h=(2 \nu / \lambda) \ln w$, with $w$ satisfying Eq. (1.3) and solved by means of the Green's function (1.5) generalized to the $d$-dimensional case. On the other hand, on the stationary submanifold defined by $\int d^{d} x \mathcal{H}_{\mathrm{B}}=0$, determining the stationary distribution $P_{\text {st }}\left(u_{n}\right)$, the orbits are given by the coupled equations (4.3) and (4.4) and will be discussed in the next section.

For later reference we also present the canonical formulation of the KPZ equation (1.17). Here we choose $q_{n} \rightarrow h(x), p_{n} \rightarrow p(x), K_{n m} \rightarrow \prod_{n} \delta\left(x_{n}-x_{n}^{\prime}\right)$, and $F_{n} \rightarrow-2\left(\nu \nabla^{2} h+(\lambda / 2) \nabla_{n} h \nabla_{n} h\right)$, and obtain action, Hamiltonian density, and equations of motion,

$$
\begin{aligned}
S_{\mathrm{KPZ}} & =\int_{0}^{T} d^{d} x d t\left(p \frac{\partial h}{\partial t}-\mathcal{H}_{\mathrm{KPZ}}\right), \\
\mathcal{H}_{\mathrm{KPZ}} & =p\left(\nu \nabla^{2} h+\frac{\lambda}{2} \nabla_{n} h \nabla_{n} h+\frac{1}{2} p\right), \\
\frac{\partial h}{\partial t} & =\nu \nabla^{2} h+\frac{\lambda}{2} \nabla_{n} h \nabla_{n} h+p, \\
\frac{\partial p}{\partial t} & =-\nu \nabla^{2} p+\lambda \nabla_{n}\left(p \nabla_{n} h\right),
\end{aligned}
$$

yielding the weak noise distribution

$$
P\left(h, T, h^{\prime}\right) \propto \exp \left[-\frac{1}{\Delta} S_{\mathrm{KPZ}}\left(h, T, h^{\prime}\right)\right],
$$

as solution of the Fokker - Planck equation

$$
\begin{aligned}
& \frac{\partial P(h, t)}{\partial t}= \\
& -\int d^{d} x \frac{\delta}{\delta h}\left[\left(\nu \nabla^{2} h+\frac{\lambda}{2} \nabla_{n} h \nabla_{n} h\right) P(h, t)\right] \\
& +\frac{\Delta}{2} \int d^{d} x d^{d} x^{\prime} \frac{\delta^{2}}{\delta h \delta h^{\prime}}\left[\prod_{n} \delta\left(x_{n}-x_{n}^{\prime}\right) P(h, t)\right] .
\end{aligned}
$$

The KPZ formulation is, however, completely equivalent to the Burgers formulation. In Eq. (4.3) only the longitudinal component of the noise field $p_{n}$ couples to the longitudinal slope field $u_{n}=\nabla_{n} h$ and we can without loss of generality assume that $p_{n}=\nabla_{n} \phi$ is purely longitudinal since Eq. (4.4) is linear in $p_{n}$; this property reflects the conserved noise $\nabla_{n} \eta$ driving the Burgers equation (1.15). Comparing Eqs (4.3) and (4.4) with Eqs. (4.10) and (4.11) we obtain complete equivalence by choosing $\nabla_{m} p_{m}=-p$ or $p=-\nabla^{2} \phi$.

\section{B. The one dimensional case}

In one dimension and focussing on the slope field $u$, which in many respects is the natural variable in discussing a growing interface, the canonical equations (4.3) and (4.4) take the simple form

$$
\begin{aligned}
& \left(\frac{\partial}{\partial t}-\lambda u \nabla\right) u=\nu \nabla^{2} u-\nabla^{2} p \\
& \left(\frac{\partial}{\partial t}-\lambda u \nabla\right) p=-\nu \nabla^{2} p
\end{aligned}
$$

originating from the Hamiltonian density

$$
\mathcal{H}_{\mathrm{B}}=p\left(\nu \nabla^{2} u+\lambda u \nabla u-\frac{1}{2} \nabla^{2} p\right) .
$$

We note that both $u$ and $p$ are scalar fields and that the $\lambda$-dependent term on the RHS of (4.4) cancels. Also, subject to the shift transformation $p=\nu(u-\varphi)$ Eqs. (4.14) and (4.15) are identical to the equations (1.10) and (1.11) discussed in paper II.

It is an important property of the one dimensional case that we can determine the explicit form of the stationary zero-energy manifold, as was the trivial case for one degree of freedom discussed in Sec. II. For

$$
p=2 \nu u
$$

the canonical equations (4.14) and (4.15) become identical and the energy density (4.16) takes the form of a total derivative, $\mathcal{H}_{\mathrm{B}} \rightarrow(2 / 3) \lambda \nu \nabla u^{3}$, yielding a vanishing total energy $E_{\mathrm{B}}=\int \mathcal{H}_{\mathrm{B}}=0$ for vanishing slope field at the boundaries. Owing to the vector character of $u_{n}$ and $p_{n}$ and the presence of the $\lambda\left(p_{n} \nabla_{m} u_{m}-p_{m} \nabla_{n} u_{m}\right)$ term in Eq. (4.4) such a transformation does not seem possible for $d>1$ and we dont have a similar contraction of the stationary submanifold.

In other words, in $d=1$ the orbit from $u^{\prime}$ to $u^{\prime \prime}$ in time $T$ for $T \rightarrow \infty$ does not only approach the zeroenergy submanifolds $p=0$ and $\nu \nabla^{2} u+\lambda u \nabla u-\frac{1}{2} \nabla^{2} p$ orthogonal to $p$ but actually converges to the submanifold $p=2 \nu u$ on the stationary submanifold. This phase space behavior is depicted in Fig. 5. In Fig. 5a we show the contraction of the stationary manifold. In Fig. 5b we depict the orbits in $p u$ phase space in a similar manner as in Fig. 2.

We finally wish to present a plausibility argument for the attraction of the orbits to the submanifold given by Eq. (4.17). Denoting the deviation from the submanifold by $\delta u$ and inserting $p=2 \nu(u+\delta u)$ in Eqs. (4.14) and (4.15) we obtain to leading order in $\delta u$,

$$
\left(\frac{\partial}{\partial t}-\lambda u \nabla\right) \delta u=\nu \nabla^{2} \delta u
$$

Noting that $\partial / \partial t-\lambda u \nabla$ in invariant under the Galilean transformation $x \rightarrow x-\lambda u_{0} t, u \rightarrow u+u_{0}$ and choosing a local frame with vanishing $u$, the Fourier modes 
$\delta u_{k} \propto \exp \left[-\nu k^{2} t\right] \rightarrow 0$ for large $t$, implying that the orbits approach the zero-energy submanifold.
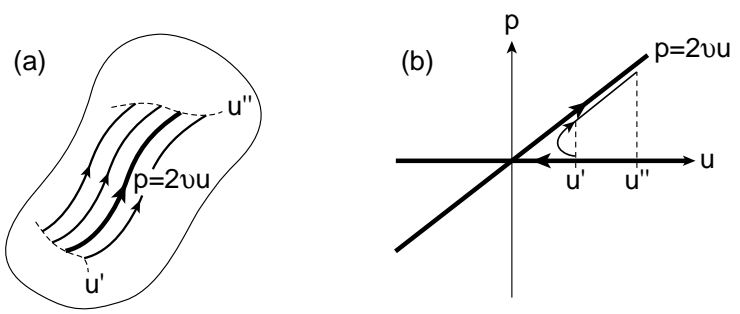

FIG. 5. Here we depict the phase space behavior in the case of the noisy one dimensional Burgers equation. In a) we show the contraction to the zero-energy submanifold $p=2 \nu u$, characteristic of the one dimensional case. In b) we show similar to Fig. 2 the orbits in $p u$ phase space.

\section{DISCUSSION AND CONCLUSION}

In this final section we derive results for the probability distributions and attempt to draw some general conclusions on the basis of the canonical phase space approach to the noisy Burgers and KPZ equations presented in the previous sections.

\section{A. The one dimensional case}

The time-dependent distribution (4.5) is determined by the form of the action $S_{\mathrm{B}}(u, T)$ in Eq. (4.1). The following analysis implies that the action has the generic form

$$
S_{\mathrm{B}}=S_{\mathrm{st}}(u)+S_{\mathrm{diff}}(u, T)+S_{\mathrm{sol}}(u, T),
$$

where $S_{\text {st }}$ yields the stationary distribution (1.9), $S_{\text {diff }}$ gives rise to corrections due to the linear diffusive modes, and $S_{\text {sol }}$ originates from the nonlinear soliton modes; both $S_{\text {diff }}$ and $S_{\text {sol }}$ must vanish in the limit $T \rightarrow \infty$ so that we attain the stationary distribution given by $S_{\text {st }}$.

In the linear Edwards-Wilkinson case for $\lambda=0$ only diffusive modes contribute and there is no growth. In wave number space the field equations (4.14) and 4.15) take the same form as Eqs. (3.8) and (3.9) in Sec. III. A straightforward generalization of Eqs. (3.10) and (3.14) then yields the orbit, $u_{k}^{\prime \prime}=u_{k}(T), u_{k}^{\prime}=u_{k}(0)$, and $\omega_{k}=\nu k^{2}$,

$$
u_{k}(t)=\frac{u_{k}^{\prime \prime} \sinh \omega_{k} t+u_{k}^{\prime} \sinh \omega_{k}(T-t)}{\sinh \omega_{k} T},
$$

and action, here $u_{k}=u_{k}(T)$,

$$
S_{\text {lin }}=\nu \int \frac{d k}{2 \pi} \frac{\left|u_{k}-u_{k}^{\prime} \exp \left(-\omega_{k} T\right)\right|^{2}}{1-\exp \left(-2 \omega_{k} T\right)}
$$

We note that in the limit $T \rightarrow \infty$ the action $S_{\text {lin }}(u, T) \rightarrow$ $\nu \int(d k / 2 \pi)\left|u_{k}\right|^{2}$ in accordance with the stationary distribution in Eq. (1.9) expanded in wave number space. Since at long times $u_{k}=\exp \left(\omega_{k} T\right) u_{k}^{\prime}$ we also find the correction

$$
S_{\text {diff }}=-\nu \int \frac{d k}{2 \pi}\left|u_{k}\right|^{2} \exp \left(-2 \omega_{k} T\right) .
$$

From Eqs. (5.3) and (5.4) we observe the simple scaling property, $u \rightarrow \mu u, S_{\text {lin }} \rightarrow \mu^{2} S_{\text {lin }}$, and $S_{\text {diff }} \rightarrow \mu^{2} S_{\text {diff }}$, i.e., scaling the slope or height field with a factor $\mu$ the action scales with $\mu^{2}$. This behavior is compatible with the equations of motion 4.14) and (4.15) provided we scale $p \rightarrow \mu p$.

As regards the time dependence of $S_{\text {lin }}$ and $S_{\text {diff }}$ we identify the crossover time

$$
T_{\mathrm{co}}^{\text {diff }} \sim \frac{1}{\nu k^{2}}
$$

depending on the wave number $k$. In the thermodynamic limit $L \rightarrow \infty$ the wave number $k$ has a continuous range and the crossover time diverges in the infrared limit $k \rightarrow 0$. Consequently, we do not have a separation of time scales.

Since the saturation width of an interface is a finite size effect time scale separation only occurs for a finite system. In the present linear case this is associated with the quantization of the wave number $k \sim 1 / L$; note that the $n=0$ mode is related to the global conservation of slope, i.e., $\int d x u$ is a constant of motion, and we have

$$
T_{\mathrm{co}}^{\mathrm{diff}} \sim \frac{L^{2}}{\nu}
$$

From the general discussion of a growing interface, $T_{\text {co }} \propto$ $L^{z}$, where $z$ is the dynamic scaling exponent, and we readily obtain $z=2$ in accordance with the diffusive mode contribution with dispersion $\omega_{k}=\nu k^{2}$. For $T \ll T_{\text {co }}^{\text {diff }}$ the diffusive modes contribute to the timedependent distribution, whereas for $T \gg T_{\mathrm{co}}^{\text {diff }}$ we cross over to the stationary regime, $S_{\text {diff }}(u, T) \rightarrow 0$, and we approach the stationary distribution (1.9) determined by $S_{\text {st }}(u)$.

\section{The stationary distribution}

In the one dimensional case the stationary distribution is known 34] and has the symmetric Gaussian form given by Eq. (1.9). This follows directly from the stationary Fokker-Planck equation (4.6) where the $\lambda$-dependent term for a Gaussian distribution becomes a total derivative and thus yields a vanishing contribution; an argument which only holds in one dimension. The slope 
field $u(x)$ is thus uncorrelated beyond a finite correlation length $\xi$ which is zero for the Burgers equation and microscopic for lattice models falling in the same universality class. The height $h(x)=\int d x u$ performs random walk yielding according to Eq. (1.12) the roughness exponent $\zeta=1 / 2$.

Within the present canonical phase space formulation the stationary distribution follows immediately from the structure $p=2 \nu u$ of the stationary submanifold. As in the harmonic oscillator case in Sec. III, the diffusive modes imply that $E_{\mathrm{B}} \rightarrow 0$ for $T \rightarrow \infty$. Thus inserting $p=2 \nu u$ in the action (4.1) in the one dimensional case and performing the time integration, we obtain in the limit $T \rightarrow \infty$

$$
S_{\mathrm{st}}(u)=\int_{0}^{\infty} d x d t p \frac{\partial u}{\partial t}=\nu \int d x u(x)^{2}
$$

and by insertion in Eq. (4.5) the distribution (1.9).

Whereas the effective fluctuation-dissipation theorem valid in one dimension implies that the stationary distribution is Gaussian and symmetric in the slope $u$ and in the height field $h$, measured relative to the mean height $\langle h\rangle$, the time-dependent distribution, converging towards the stationary one, is expected to exhibit an asymmetric shape corresponding to the predominance of peaks in $h$ in the growth direction.

\section{The long-time skew distribution}

The phase space approach also allows us to estimate the long-time corrections to the stationary distribution. Following the reasoning in Sec. III we replace for large $T$ the orbit near the submanifold $p=2 \nu u$ with an orbit on the submanifold. Inserting $p=2 \nu u$ in Eq. (4.14) the orbits on the stationary submanifold are governed by the noiseless Burgers equation with $\nu$ replaced by $-\nu$,

$$
\left(\frac{\partial u}{\partial t}-\lambda u \nabla\right) u=-\nu \nabla^{2} u
$$

This equation is readily solved by means of the Cole-Hopf transformation (1.2) with solution given by Eqs. (1.4-1.6) with $-\nu$ substituted for $\nu$. For the action (4.1) we then obtain $S_{\mathrm{B}}=\nu \int d x\left[u^{2}-u^{\prime 2}\right]$ and for the time-dependent probability distribution

$$
P(u, T) \propto P_{\text {st }}(u) P_{\text {skew }}(u, T),
$$

where the symmetric stationary distribution $P_{\mathrm{st}}(u)$ is given by Eq. (1.9) and the time-dependent skew correction by

$$
P_{\text {skew }}(u, T)=\exp \left[\frac{\nu}{\Delta} \int d x u^{\prime}(x)^{2}\right],
$$

with $u^{\prime}=\nabla h^{\prime}$ and $u=\nabla h$ related by the Cole-Hopf transformation $\exp \left[-\frac{\lambda}{2 \nu} h^{\prime}(x)\right]=\int d x^{\prime} G\left(x-x^{\prime}, T\right) \exp \left[-\frac{\lambda}{2 \nu} h\left(x^{\prime}\right)\right]$.

Note that since $\int d x^{\prime} G=1$ the correction $u^{\prime 2}$ vanishes in the limit $T \rightarrow \infty$ and $P_{\text {skew }} \rightarrow 1$.

In order to examine the skewness of the distribution it is convenient to eliminate the stationary component by forming the ratio

$$
\frac{P(u, T)}{P(-u, T)}=\exp \left[\frac{\nu}{\Delta} \int d x\left(u_{+}^{\prime 2}-u_{-}^{\prime 2}\right)\right],
$$

where according to the Cole-Hopf transformation (5.11)

$$
\exp \left[-\frac{\lambda}{2 \nu} h_{ \pm}^{\prime}\right]=\int d x^{\prime} G(x-x, T) \exp \left[\mp \frac{\lambda}{2 \nu} h\right] .
$$

Inserting the Green's function (1.5), $G(x, T)=$ $[4 \pi \nu T]^{-1 / 2} \exp \left[-x^{2} / 4 \nu T\right]$, we consider first a few simple cases.

For a constant slope $u=u_{0}$, i.e., $h=u_{0} x-h_{0}$, we obtain, performing the Gaussian integration, $h_{ \pm}^{\prime}=$ $\pm\left(u_{0} x+h_{0}\right)-\lambda T u_{0}^{2} / 2$. We note that the growth term $\lambda u \nabla u$ as expected gives rise to a time-dependent term in $h_{ \pm}^{\prime}$. This term, however, is compensated for by transforming to a co-moving frame as in the KPZ equation (1.17). The slope $u^{\prime}$, however, is independent of $T$ and we obtain $u_{ \pm}^{\prime}= \pm u_{0}$, yielding $S_{\mathrm{B}}=0$ and $P=$ const., i.e., no dynamics. This is consistent with the fact that $u=u_{0}$ and $p=p_{0}$ trivially satisfy the field equations (4.14) and (4.15) yielding $E_{\mathrm{B}}=0$, and thus corresponds to a stationary state, as also discussed in paper II.

Choosing a slope depending linearly on $x, u=2 s_{0} x$, corresponding to a parabolic height profile, $h=s_{0} x^{2}+h_{0}$, we obtain $u_{ \pm}^{\prime}= \pm 2 s_{0} x /\left(1 \pm 2 \lambda T s_{0}\right)$, yielding the skewness ratio

$$
\frac{P(u, T)}{P(-u, T)}=\exp \left[-\frac{32}{3} \frac{\nu \lambda}{\Delta} s_{0}^{3} \frac{T L^{3}}{\left(1-\left(2 \lambda T s_{0}\right)^{2}\right)^{2}}\right],
$$

where we have introduced the size $L$ of the system. The expression (5.14) only holds for $\lambda T s_{0}<1$, the important aspect is, however, the dependence on the sign of $s_{0}$, i.e., the slope of the slope or the bias of the height profile. For $s_{0}>0$ corresponding to a parabolic shape of $h$ with a minimum, i.e., a downward peak, $P(u, T) / P(-u, T)<1$, whereas for $s_{0}<0$, yielding an upward peak in $h$, we have $P(u, T) / P(-u, T)>1$. This behavior implies that the distribution is skew at finite times and that the upward peaks in $h$ statistically are more pronounced than the downward peaks, i.e., the distribution is biased and changes asymmetrically towards the symmetric stationary distribution.

We can also gain some insight in the inviscid limit $\nu \rightarrow 0$ from a saddle point calculation along the lines of similar treatments of the noiseless Burgers equation 
32,33. From Eq. (5.11) we obtain, inserting the Green's function (1.5),

$$
\exp \left[-\frac{\lambda}{2 \nu} h_{ \pm}^{\prime}\right]=\int d x^{\prime}[4 \pi \nu T]^{-1 / 2} \exp \left[-(1 / 2 \nu) \phi_{ \pm}\right]
$$

where $\phi_{ \pm}\left(x, x^{\prime}\right)=\left(x-x^{\prime}\right)^{2} / 2 T \pm \lambda h\left(x^{\prime}\right)$. In the limit $\nu \rightarrow 0$ the integral is dominated by the local minima given by $d \phi_{ \pm}\left(x, x^{\prime}\right) / d x^{\prime}=0$ and the condition $d^{2} \phi_{ \pm}\left(x, x^{\prime}\right) / d x^{\prime 2}>0$. The solutions $x_{ \pm}^{\prime}$ are thus determined by the implicit equation $x_{ \pm}^{\prime}-x=\mp \lambda T u\left(x_{ \pm}^{\prime}\right)$ together with the conditions $\pm\left(d u / d x^{\prime}\right)_{x^{\prime}=x_{ \pm}^{\prime}}>-1 / \lambda T$, and we obtain the ratio

$$
\frac{P(u, T)}{P(-u, T)}=\exp \left[\frac{\nu}{\Delta} \int d x \frac{\left(x-x_{+}^{\prime}\right)^{2}-\left(x-x_{-}^{\prime}\right)^{2}}{(\lambda T)^{2}}\right],
$$

which can be used in order to analyze the skewness of various profiles $u(x)$. Note that $P(u, T) / P(-u, T) \rightarrow 1$ in the limit $T \rightarrow \infty$, corresponding to vanishing skewness. In Fig. 6 we have depicted the saddle point construction.

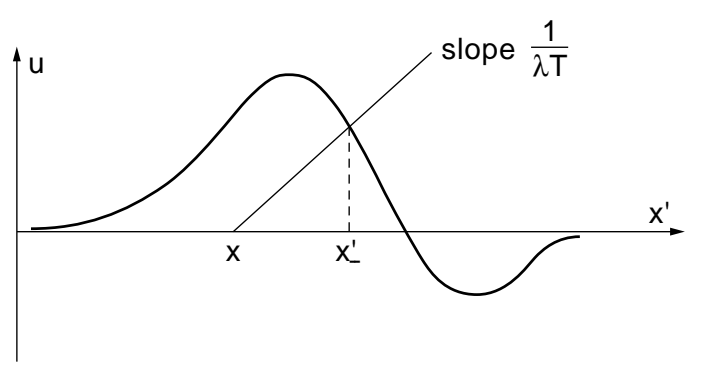

FIG. 6. Here we show the saddle point construction valid in the inviscid limit $\nu \rightarrow 0$. The saddle point condition $x_{-}^{\prime}-x=\lambda T u\left(x_{-}^{\prime}\right)$ determines $x_{-}^{\prime}$ as the intersection between the line with slope $1 / \lambda T$ and the slope profile $u\left(x^{\prime}\right)$. The intersection of the line with the $x^{\prime}$ axis determines $x$.

More insight into the dynamics underlying the skew long-time distribution is gained by expanding (5.11) in the nonlinear coupling. Choosing a compact notation, $u_{x}=u(x), h_{x}=h(x), G_{x x^{\prime}}(T)=G\left(x-x^{\prime}, T\right)$, and $\delta_{x x^{\prime}}=\delta\left(x-x^{\prime}\right)$, we obtain to leading order in $\lambda$

$$
\begin{aligned}
u_{x}^{\prime} & =\int d x^{\prime} G_{x x^{\prime}}(T) u_{x^{\prime}} \\
& -(\lambda / \nu) \int d x^{\prime} G_{x x^{\prime}}(T) u_{x^{\prime}} h_{x^{\prime}} \\
& +(\lambda / \nu) \int d x^{\prime} d x^{\prime \prime} G_{x x^{\prime}}(T) G_{x x^{\prime \prime}}(T) u_{x^{\prime}} h_{x^{\prime \prime}} .
\end{aligned}
$$

Correspondingly, $P_{\text {skew }}$ factorizes in a component independent of $\lambda$ and a component depending on $\lambda$,

$$
\begin{aligned}
& P_{\text {skew }}=P_{0} P_{\lambda} \\
& P_{0}=\exp \left[\frac{\nu}{\Delta} \int d x d x^{\prime} G_{x x^{\prime}}(2 T) u_{x} u_{x^{\prime}}\right] \\
& P_{\lambda}=\exp \left[-\frac{2 \lambda}{\Delta} \int d x d x^{\prime} d x^{\prime \prime} F_{x x^{\prime}, x^{\prime} x^{\prime \prime}}(T) u_{x} u_{x^{\prime}} h_{x^{\prime \prime}}\right]
\end{aligned}
$$

Here the kernel $F$ is given by

$$
\begin{aligned}
& F_{x x^{\prime}, x^{\prime} x^{\prime \prime}}(T)= \\
& G_{x x^{\prime}}(2 T) \delta_{x^{\prime} x^{\prime \prime}}-\int d y G_{y x}(T) G_{y x^{\prime}}(T) G_{y x^{\prime \prime}}(T) .
\end{aligned}
$$

For vanishing $\lambda$ only $P_{0}$ contributes. In wave number space we thus obtain, introducing $u_{k}=$ $\int d x \exp (i k x) u(x)$ and noting from Eq. (1.5) that $G_{k}(t)=\exp \left[-\nu k^{2} t\right]$, the distribution

$$
P\left(u_{k}, T\right) \propto \exp \left[-\frac{\nu}{\Delta} \int \frac{d k}{2 \pi}\left|u_{k}\right|^{2}\left[1-\exp \left(-2 \nu k^{2} T\right)\right]\right] .
$$

This result is completely equivalent to Eq. (3.16) for the damped oscillator discussed in Sec. III. For $\lambda=0$, corresponding to the Edwards Wilkinson case, only gapless diffusive modes $u_{k} \propto \exp \left[-\nu k^{2} t\right]$ contribute to the timedependent distribution; we also note that the distribution remains symmetric.

To first order in $\lambda$ the diffusive modes interact and we obtain a correction to $P_{\text {skew }}$ given by $P_{\lambda}$ in Eq. (5.20). This contribution is odd in $u$ and characterized by the kernel $F$. In other words, the scattering of the diffusive modes on one another due to the term $\lambda u \nabla u$ in the Burgers equation (5.8) yields a skew distribution in $u$. In the limit $T \rightarrow \infty$ this term vanishes and we obtain the symmetric stationary distribution. Correspondingly, in wave number space we have to order $\lambda$

$$
\begin{aligned}
& P_{\lambda}\left(u_{k}, T\right) \propto\left[-\frac{2 \lambda}{\Delta} \int \frac{d k}{2 \pi} \frac{d k^{\prime}}{2 \pi} F_{k, k^{\prime}}(T) u_{k} u_{-k-k^{\prime}} h_{k^{\prime}}\right], \\
& F_{k, k^{\prime}}(T)=G_{k}(2 T)-G_{k}(T) G_{k+k^{\prime}}(T) G_{k^{\prime}}(T),
\end{aligned}
$$

showing the interaction between the various $k$-modes (the cascade).

\section{The short-time skew distribution}

At shorter times, i.e., $T \leq T_{\mathrm{co}}^{\text {diff }}$, the approximation of replacing the orbit near the zero-energy submanifold with an orbit on the submanifold ceases to be valid and we have to consider the equations of motions (4.14) and 
(4.15) in more detail in order to identify the contribution to $S_{\text {sol }}$.

Although the noiseless Burgers equation is exactly soluble by means of the Cole-Hopf transformation, the equations of motion (4.14) and (4.15) describing the noisy case are presumably not exactly integrable. They do, however, admit special permanent profile or solitary wave solutions with superposed linear diffusive modes. It moreover follows from the path integral formulation in paper II that an arbitrary interface profile can be represented by a dilute gas of solitons, at least in the inviscid limit for small $\nu$, where we can neglect soliton overlap contributions.

In the form given by Eqs. (1.10) and (1.11) these equations were discussed in detail in paper II where we identified the elementary excitations. The spectrum consists of right hand and left hand nonlinear soliton modes with superposed linear diffusive modes. The localized soliton modes have a finite energy and thus correspond to the nearby phase space orbits approaching the zero-energy manifold. At long times the soliton energy must go to zero and the remaining superposed diffusive modes determine $P_{\text {skew }}$ as discussed above. Note that the nonlinear soliton mode can be regarded as a bound state of diffusive modes; this follows from the stability analysis in paper I and is a consequence of Levinson's theorem. In paper II we performed a shift transformation of the noise variable, $p \rightarrow \nu(u-\varphi)$, in order to express the Hamiltonian density $\mathcal{H}_{\mathrm{B}}$ in Eq. (4.16) in a canonical form with a harmonic component describing the linear case, yielding the field equations (1.10) and (1.11). In the present context we summarize the soliton dynamics in terms of $p$ and $u$ in accordance with the present interpretation of the transition to the stationary state. The right and left hand solitons then play a different role in the weight of the interface morphology. In the static limit the soliton modes have the form

$$
u(x)= \pm u_{0} \tanh \left[\frac{\lambda u_{0}}{2 \nu}\left(x-x_{0}\right)\right],
$$

with amplitude $u_{0}$ and position $x_{0}$. Using the Galilean invariance of the field equations (4.14) and (4.15), i.e., observing that the operator $\partial / \partial t-\lambda u \nabla$ is invariant under the transformation $x \rightarrow x-\lambda \tilde{u} t, u \rightarrow u+\tilde{u}$, propagating solitons with boundary values $u \rightarrow u_{ \pm}$for $x \rightarrow \pm L, L$ is the size of the system, are obtained by boosting the static solitons in Eq. (5.25). Moreover, the propagation velocity $v$ is given by $u_{+}$and $u_{-}$according to the soliton condition

$$
u_{+}+u_{-}=-\frac{2 v}{\lambda}
$$

which thus determines the kinetics and matching conditions for a multi-soliton configuration describing a growing interface.

The right hand soliton corresponds to $p=0$ and is according to Eq. (4.14) a solution of the noiseless Burgers equation (1.1). Dynamical attributes are a feature of the noisy case and this soliton thus carries vanishing energy, $E_{\mathrm{B}}=\int d x \mathcal{H}_{\mathrm{B}}=0$, vanishing momentum, $\Pi_{\mathrm{B}}=\int d x u \nabla p=0$, and vanishing action $S_{\mathrm{B}}=0$, and corresponds to an orbit on the transient zero-energy manifold. We note that a single right hand soliton or a multisoliton solution cannot satisfy the boundary condition of vanishing slope.

The left hand soliton moves on the submanifold $p=$ $2 \nu u$ and satisfies according to Eq. (4.14) the noiseless Burgers equation (1.1) with $\nu$ replaced by $-\nu$. It carries energy, momentum, and action given by

$$
\begin{aligned}
E_{\mathrm{B}} & =\frac{2}{3} \nu \lambda\left(u_{+}^{3}-u_{-}^{3}\right), \\
\Pi_{\mathrm{B}} & =\nu\left(u_{+}^{2}-u_{-}^{2}\right), \\
S_{\mathrm{B}} & =\frac{1}{6} \nu \lambda T\left|u_{+}-u_{-}\right|^{3} .
\end{aligned}
$$

Since $u_{+}<u_{-}$for a right hand soliton $E_{\mathrm{B}}$ is negative and $\Pi_{\mathrm{B}}$ has according to Eq. (5.26) the same sign as the velocity $v$. The action $S_{\mathrm{B}}$ is positive and Galilean invariant. We also note that although the soliton is confined to the submanifold $p=2 \nu u$ the energy is nonvanishing. This is associated with the nonequal boundary values $u_{+}$ and $u_{-}$and also follows directly from Eq. (4.16), where inserting $p=2 \nu u$ we obtain a total derivative. Hence, $E_{\mathrm{B}}=\int d x \mathcal{H}_{\mathrm{B}}=(2 \nu \lambda / 3)\left(u_{+}^{3}-u_{-}^{3}\right)$, where $u_{+}$and $u_{-}$ are the boundary values.

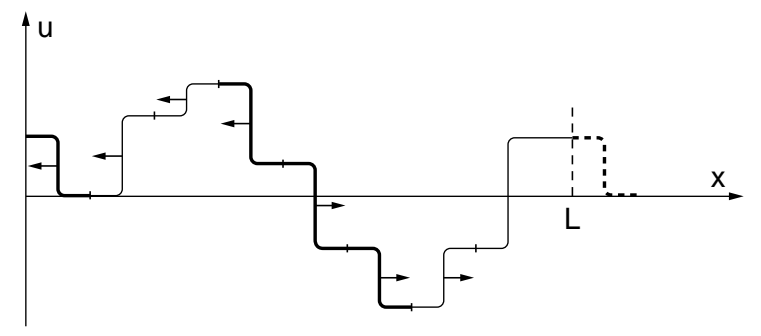

FIG. 7. We depict the slope soliton morphology for a growing interface in a system of size $L$. In accordance with a growth situation we have imposed periodic boundary conditions. The left hand solitons carrying energy, momentum, and action are indicated by solid lines.

As discussed in paper II the morphology of a growing interface is determined by matching a set of right hand and left hand solitons according to the soliton condition (5.26). The right and left hand solitons are exact solutions of the damped and undamped $(-\nu)$ noiseless Burgers equations, respectively. Their stability is associated with the nonequality of the boundary values, 
$u_{+} \neq u_{-}$, corresponding to a nonvanishing slope current at the boundaries. In a multi-soliton configuration with vanishing boundary conditions current thus flows between the solitons. The current is generated by the left hand solitons and dissipated by the right hand solitons - this is another view of the cascade driving the noisy Burgers equation. The morphology is depicted in Fig. 7 .

The probability of a soliton morphology is determined by $S_{\text {sol }}(u, T)$. Assuming that $S_{\text {sol }}=\lambda F(u, T)$ a scaling argument similar to linear case, $u \rightarrow \mu u, p \rightarrow \mu p$, $\lambda \rightarrow \mu^{-1} \lambda$, and $S_{\text {sol }} \rightarrow \mu^{2} S_{\text {sol }}$, following from the general form of the equations of motion (4.14) and (4.15) and the action (4.1) implies that $F \rightarrow \mu^{3} F$. This is consistent with the expression (5.29) and we obtain

$$
S_{\mathrm{sol}}(u, T)=\frac{1}{6} \nu \lambda T \sum_{\mathrm{lhs}}\left|u_{+}-u_{-}\right|^{3},
$$

where the summation is only over contributing left hand solitons (lhs).

Beyond this point our discussion becomes necessarily more qualitative and heuristic since we dont possess a complete solution of the coupled field equations. The non-integrability and the constraint imposed by the soliton condition (5.26) imply that we only have available a dilute gas of right hand and left hand solitons. First we notice that for an infinite system the soliton velocity $v$ given by Eq. (5.26) ranges freely, implying that the center of mass of the soliton amplitude $u_{\mathrm{cm}}=\left(u_{+}+u_{-}\right) / 2$ also can take arbitrary values. Since $T$ enters as a prefactor in Eq. (5.30) $S_{\text {sol }}$ grows for $T \rightarrow \infty$, i.e., $P \propto \exp \left[-S_{\mathrm{sol}} / \Delta\right] \rightarrow 0$, and we are unable to identify a solitonic crossover time. This is consistent with the general discussion of a growing interface [37]. Generally, the crossover time $T_{\text {co }} \propto L^{z}$, where $z$ is the dynamic exponent. As in the linear case for $\lambda=0$ discussed above the saturation width for a growing interface is a finite size effect and the transient growth does not saturate to stationary growth for an infinite system. Whereas the situation was easy to analyze in the linear case where we can identify the independent modes, and where the system size $L$ is replaced by the inverse wave number $1 / k$, i.e., the thermodynamic limit is probed in the infrared limit $k \rightarrow 0$, the situation is more subtle in the nonlinear soliton case since we dont have a normal mode structure but only approximate elementary excitations.

On the other hand, for a finite size system, imposing for example periodic boundary conditions as indicated in Fig. 7 in order to ensure a growing interface in $h$ as the slope solitons revolve, the velocity $v$ becomes endowed with a scale and is quantized in units of $L / T$. Notice here the important difference between the diffusive case and the solitonic case. In the diffusive case the excitations are not propagating but are linear combinations of growing and decaying modes as discussed in Sec. III, and the system size $L$ only enters in the quantization of the wave number $k \propto 1 / L$, yielding the crossover time $T_{\mathrm{co}}^{\text {diff }} \propto L^{2}$. In the solitonic case the localized modes are propagat- ing giving rise to genuine nonequilibrium growth. The system size $L$ then enters together with the time $T$ in setting a scale for the velocity $v$.

A simple estimate, replacing the soliton amplitude $u=u_{+}-u_{-}$in the general expression (5.30) by $u_{\mathrm{cm}}=$ $\left(u_{+}+u_{-}\right) / 2=-v / \lambda$ from Eq. (5.26) and moreover $v$ by $L / T$ we obtain $S_{\text {sol }} \propto$ const. $\nu L^{3} / \lambda^{2} T^{2}$ which inserted in $P \propto \exp \left[-S_{\text {sol }} / \Delta\right]$ yields the soliton crossover time

$$
T_{\mathrm{co}}^{\mathrm{sol}} \propto\left(\frac{\nu}{\Delta}\right) \frac{1}{\lambda} L^{3 / 2},
$$

and we infer the dynamic exponent $z=3 / 2$. In the transient short-time regime $T \ll T_{\text {co }}^{\text {sol }}$ the soliton configurations contribute to $P$; in the long-time regime $T \gg T_{\mathrm{co}}^{\mathrm{sol}}$ the soliton contribution vanishes and only the diffusive modes and their interactions contribute to $P$. We also notice that the expression (5.31) is consistent with the dimensionless argument $\lambda(\Delta / \nu)^{1 / 2} t / x^{3 / 2}$ in the scaling function for the slope correlation function discussed in paper II and in 43, 44, 52]. In Fig. 8 we have depicted the crossover regimes.

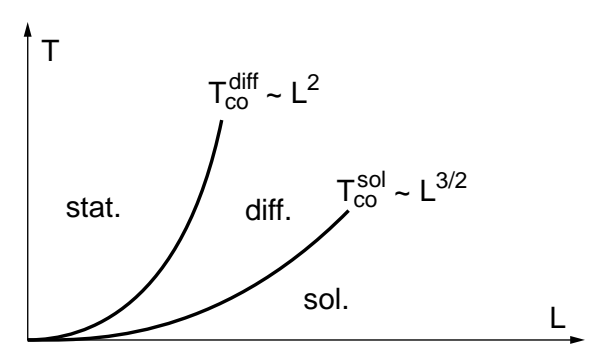

FIG. 8. Here we depict the crossover time as a function of the system size $L$. In the early time regime for $T \ll T_{\text {co }}^{\text {sol }}$ the distribution is dominated by soliton contributions. In the intermediate time regime for $T_{\mathrm{co}}^{\text {diff }} \gg T \gg T_{\mathrm{co}}^{\mathrm{sol}}$ the soliton contributions become suppressed leaving the diffusive mode contributions. Finally, for $T \gg T_{\text {co }}^{\text {diff }}$ the diffusive modes also die out and we approach the stationary distribution.

The short-time probability distribution $P(h, T)$ for $T \ll T_{\mathrm{co}}^{\mathrm{sol}}$ has been discussed within the directed polymer approach 331,66]. Based upon a replica scaling analysis [67] one finds for positive $h$ (measured relative to the growing mean height) the heuristic expression $P(h, T) \propto \exp \left[-\left(h / T^{1 / 3}\right)^{\eta}\right]$, where $\eta=3 / 2$; for $h<0$ based on numerical results, $\eta \sim 2.5$. Recent exact results for the asymmetric exclusion model which falls in the same universality class as the Burgers equation, see ref. [68], where other references also can be found, also seems to have bearings on the height distribution. Using the Bethe ansatz method a skew distribution, characterized by the exponents $3 / 2$ and $5 / 2$, have been found for the 
large deviation function of the time averaged current.

Within the present soliton approach we can derive a qualitative expression for the early time height distribution by noting that $\left|u_{+}-u_{-}\right|^{3} \sim(u L)^{3 / 2}(T \lambda)^{-3 / 2} \sim$ $h^{3 / 2}(T \lambda)^{-3 / 2}$. Inserting this result in the general expression (5.30) we obtain

$$
P_{\text {sol }}(h, T) \propto \exp \left[-\frac{\nu}{\Delta}\left[\frac{1}{\lambda T}\right]^{1 / 2} h^{3 / 2}\right],
$$

in accordance with the directed polymer-replica based result and related to the exact results for the asymmetric exclusion model. The skewness of the distribution in $h$ must then arise from the bias in the statistical weight $\exp [-S / \Delta]$ assigned to the left and right hand solitons giving rise to a predominance of right hand solitons $(S=0)$, corresponding to relative forward growth. Unfortunately, our present understanding of the soliton approach and the inaccessibility of a more detailed multisoliton solution do not allow a more detailed analysis.

\section{B. The general case}

In the general case for $d>1$ the slope and noise fields $u_{n}$ and $p_{n}$ have longitudinal vector character and are governed by Eqs. (4.3) and (4.4) determining an orbit $\left(u_{n}\left(x_{p}, t\right), p_{n}\left(x_{p}, t\right)\right)$ in $p_{n} u_{n}$ phase space. At long times the orbit must pass close to the stationary saddle point $\left(u_{n}, p_{n}\right)=(0,0)$ in order to induce Markovian behavior and then progress onto the stationary zeroenergy manifold $E_{\mathrm{B}}=\int d^{d} x \mathcal{H}_{\mathrm{B}}=0$ with energy density given by Eq. (4.2), yielding the weak noise distribution $P \propto \exp \left[-S_{\mathrm{B}} / \Delta\right]$ with $S_{\mathrm{B}}$ given by Eq. (4.1).

In $d=1$, as discussed in Sec. A, the orbit on the stationary manifold is attracted to the submanifold $p=2 \nu u$, yielding the symmetric stationary distribution (1.9) with time-dependent skew corrections. For $d>1$ this behavior is only encountered in the linear Edwards-Wilkinson case for $\lambda=0$; note also the general discussion in Sec. II. The attraction to the submanifold $p_{n}=2 \nu u_{n}$ is associated with the underlying fluctuation-dissipation theorem and gives rise to the stationary Gaussian distribution $P_{\mathrm{st}} \propto \exp \left[-(\nu / \Delta) \int d^{d} x u_{n}(x)^{2}\right]$; the corresponding free energy is $F=(1 / 2) \int d^{d} x u_{n}(x)^{2}$. This distribution yields the roughness exponent $\zeta=(2-d) / 2$. The dynamic exponent $z=2$, corresponding to the diffusive mode contribution; note that the Galilean invariance is not operative in the linear case and that we consequently dont have the scaling law constraint $\zeta+z=2$.

In the nonlinear case $\lambda \neq 0$ for $d>2$ the long-time orbit emerging from the vicinity of the stationary point $\left(u_{n}, p_{n}\right)=(0,0)$ diverges for larger $u_{n}$ from the submanifold $p_{n}=2 \nu u_{n}$ which constitutes a sort of tangent plane to the zero-energy surface at the stationary point. In the limit $\lambda \rightarrow 0$ the energy surface then collapses to the tangent plane. More detailed, in the stationary limit the distribution is given by

$$
P_{\text {st }} \propto \exp \left[-\frac{1}{\Delta} \int_{0}^{\infty} d^{d} x d t p_{n} \frac{\partial u_{n}}{\partial t}\right] .
$$

Noting that $p_{n}$ is slaved to $u_{n}$ on the orbit we obtain in general (to leading order in $\nabla_{n}$ )

$$
p_{n}=u_{n} F_{d}\left(u^{2}\right)+u_{p} \nabla_{n} u_{p} G_{d}\left(u^{2}\right),
$$

where the scalar functions $F_{d}$ and $G_{d}$ depend on the invariant $u^{2}=u_{n} u_{n}$ and parametrically on the dimension $d$. In one dimension we have $F_{1}=2 \nu$ and $G_{1}=0$. For $d>1$ to leading order in $\nabla_{n}$ the distribution $P_{\text {st }}$ is even in $u_{n}$. Note, however, that for $G_{d} \neq 0$ there is a skew correction to $P_{\mathrm{st}}$. Assuming that this analysis is valid, the determination of $F_{d}$ and $G_{d}$ is then given by the solution of the equations of motion (4.3) and (4.4) on the stationary zero-energy manifold.

In the nonlinear case for $d>2$ the rough phase governed by the strong coupling fixed point only appears for a renormalized coupling strength $\tilde{\lambda}=(\Delta \lambda)^{2} / \nu^{3}$ exceeding a finite threshold value $\tilde{\lambda}_{c}$ 69 71 and recent work [55.56] moreover indicates that unlike the case in $d=1$ even the stationary probability distribution exhibits skewness, i.e., $P_{\mathrm{st}}(u) \neq P_{\mathrm{st}}(-u)$. Such a behavior does not seem compatible with the analysis above and indicates that the present weak noise approach only applies to the weak coupling phase for $d>2$. Presumably, the strong coupling phase for $\tilde{\lambda}>\tilde{\lambda}_{c}$ is only accessed beyond a critical noise strength $\Delta_{c}, \tilde{\lambda}_{c}=\left(\Delta_{c} \lambda\right)^{2} / \nu^{3}$. These issues and the fact that the strong coupling fixed point (fortuitously) can be analysed in $d=1$ in a nonperturbative weak noise approximation remains not very well understood and call for further investigations.

\section{Summary and conclusion}

In the present paper we have advanced a general weak noise canonical phase space approach to stochastic systems governed by a Langevin equation driven by additive white noise. Reformulating the associated FokkerPlanck equation in the nonperturbative weak noise limit in terms of a Hamilton-Jacobi equation we have discussed the time-dependent and stationary probability distributions from a canonical phase space point of view. The issue of solving the stochastic Langevin equation or the associated Fokker-Planck equation is thus replaced by solving coupled Hamilton equations of motion determining the orbits in phase space. The stochastic nature of the underlying problem is reflected in a peculiar topology of the energy surfaces different from the one encountered in ordinary dynamical problems. The Markovian behavior thus corresponds to the existence of a stationary hyperbolic saddle point which controls the behavior of the orbits in the long time limit.

We have in particular applied the canonical phase space approach to the noisy Burgers equation describing 
a growing interface in one dimension. We have recovered the well-known stationary distribution and derived expressions for the time-dependent distribution, at long times governed by linear diffusive modes and their interaction and at shorter time by nonlinear soliton excitations. In higher dimensions where the noisy Burgers equation predicts a kinetic phase transition to a strong coupling phase the canonical phase space approach only seems to access the weak coupling phase.

\section{ACKNOWLEDGMENTS}

Discussions with M. Kosterlitz, B. Derrida, T. Hwa, J. Hertz, P. Cvitanović, T. Bohr, M. H. Jensen, J.P. Bouchaud, J. Krug, M. Alava, K. B. Lauritsen, K. Mølmer and A. Svane are gratefully acknowledged.

[1] H. C. Fogedby, cond-mat/9812091.

[2] H. C. Fogedby, Phys. Rev. E 57, 2331 (1998), denoted I.

[3] H. C. Fogedby, Phys. Rev. E 57, 4943 (1998), denoted II.

[4] H. C. Fogedby, Phys. Rev. Lett. 80, 1126 (1998).

[5] J.M.Burgers, Proc. Roy. Neth. Acad. Soc. 32, 414,643,818 (1929).

[6] J. Burgers, The Nonlinear Diffusion Equation (Riedel, Boston, 1974).

[7] D. Forster, D. R. Nelson, and M. J. Stephen, Phys. Rev. Lett. 36, 867 (1976).

[8] D. Forster, D. R. Nelson, and M. J. Stephen, Phys. Rev. A 16, 732 (1977).

[9] P.C.Martin, E.D.Siggia, and H.A.Rose, Phys. Rev. A 8, 423 (1973).

[10] C. de Dominicis, Phys. Rev. E 12, 567 (1975).

[11] C. de Dominicis, J. Phys. (Paris), Colloq. 37, 247 (1976).

[12] R.Baussch, H.K.Janssen, and H.Wagner, Z. Phys. B 24, 113 (1976).

[13] H. K. Janssen, Z. Phys. B 23, 377 (1976).

[14] C. D. Dominicis and L. Peliti, Phys. Rev. B 18, 353 (1978).

[15] S.-K. Ma, Modern theory of critical phenomena (Benjamin Press, London, 1976).

[16] P. M. Chaikin and T. C. Lubensky, Principles of condensed matter physics (Cambridge University Press, Cambridge, 1995).

[17] P. S. in, Topics in Nonlinear Physics, ed. N.J. Zabusky (Springer, New York, 1968).

[18] E. Jackson, Perspectives of nonlinear dynamics (Cambridge University Press, Cambridge, 1990).

[19] G. B. Whitham, Nonlinear Waves (Wiley, New York, 1974).

[20] L. Landau and E. Lifshitz, Fluid Mechanics (Pergamon Press, Oxford, 1959).

[21] S. Kida, J. Fluid. Mech. 93, 337 (1979).
[22] S. Gurbatov and A. Saichev, Sov. Phys. JETP 53, 347 (1981).

[23] E. Aurell et al., J. Fluid. Mech. 238, 467 (1992).

[24] Z. S.She, E. Aurell, and U. Frisch, Comm. Math. Phys. 148, 623 (1992).

[25] S. E. Episov and T. J. Newman, Phys. Rev. E 48, 1046 (1993).

[26] S. E. Episov, Phys. Rev. E 49, 2070 (1994).

[27] T. J. Newman, Phys. Rev. E 55, 6989 (1997).

[28] A. Scott, F. Y. F. Chu, and D. W. McLaughlin, Proc. IEEE 61, 1443 (1973).

[29] J.D.Cole, Quart. Appl. Math. 9, 22 (1951).

[30] E. Hopf, Comm. Pure Appl. Math. 3, 201 (1950).

[31] T. Halpin-Healy and Y. C. Zhang, Phys. Rep. 254, 215 (1995).

[32] M. Kardar, G. Parisi, and Y. C. Zhang, Phys. Rev. Lett. 56, 889 (1986).

[33] E. Medina, T. Hwa, M. Kardar, and Y. C. Zhang, Phys. Rev. A 39, 3053 (1989).

[34] D. A. Huse, C. L. Henley, and D. S. Fisher, Phys. Rev. Lett. 55, 2924 (1985).

[35] J. Krug and H. Spohn, Solids Far from Equilibrium; Kinetic roughening of growing surfaces: Fractal Concepts in Surface Growth (Cambridge University Press, Cambridge, 1992).

[36] J. Krug, Adv. Phys. 46, 139 (1997).

[37] A. L. Barabasi and H. E. Stanley, Fractal Concepts in Surface Growth (Cambridge University Press, Cambridge, 1995).

[38] F. Family and T. Vicsek, J. Phys. A 18, L75 (1985).

[39] R. Jullien and R. Botet, J. Phys. A 18, 2279 (1985).

[40] S. F. Edwards and D. R. Wilkinson, Proc. Roy. Soc. London A 381, 17 (1982).

[41] H. C. Fogedby, Phys. Rev. Lett. 73, 2517 (1994).

[42] H. C. Fogedby, Phys. Rev. E 58, 1690 (1998).

[43] T. Hwa and E. Frey, Phys. Rev. A 44, R7873 (1991).

[44] E. Frey, U. C. Täuber, and T. Hwa, Phys. Rev. E 53, 4424 (1996).

[45] T. Hwa, Phys. Rev. Lett. 69, 1552 (1992).

[46] E. Frey and U. C. Täuber, Phys. Rev. E 50, 1024 (1994).

[47] U. C. Täuber and E. Frey, Phys. Rev. E 51, 6319 (1995).

[48] D. Dahr, Phase Transitions 9, 51 (1987).

[49] L. H. Gwa and H. Spohn, Phys. Rev. Lett. 68, 725 (1992).

[50] L. H. Gwa and H. Spohn, Phys. Rev. A 46, 844 (1992).

[51] H. van Beijeren, R. Kutner, , and H. Spohn, Phys. Rev. Lett. 54, 2026 (1985).

[52] H. Janssen and B. Schmittman, Z. Phys. B 63, 517 (1986).

[53] T. M. Ligget, Interacting Particle Systems (SpringerVerlag, Berlin, 1985).

[54] M. Lässig, Nucl. Phys. B 448, 559 (1995).

[55] M. Lässig, Phys. Rev. Lett. 80, 2366 (1998).

[56] C.-S. Chin and M. den Nijs, cond-mat/9810083.

[57] J. Zinn-Justin, Quantum Field Theory and Critical Phenomena (Oxford University Press, Oxford, 1989).

[58] H. Risken, The Fokker-Planck Equation (SpringerVerlag, Berlin, 1989).

[59] H. C. Fogedby, Theoretical Aspects of Mainly Low Dimensional Magnetic Systems (Springer-Verlag, New York, 1980). 
[60] L. Landau and E. Lifshitz, Mechanics (Pergamon Press, Oxford, 1959).

[61] H. Goldstein, Classical Mechanics (Addison-Wesley Publishing Company, Inc., Reading, Massachusetts, 1980).

[62] V. Arnold, Mathematical Methods of Classical Mechanics (Springer-Verlag, New York, 1983).

[63] E. Ott, Chaos in Dynamical Systems (Cambridge University Press, Cambridge, 1993).

[64] A. Das, Field Theory - A Path Integral Approach (World Scientific, Singapore, 1993).

[65] R. Feynman and A. Hibbs, Quantum Mechanics and Path
Integrals (McGraw-Hill Book Co., New York, 1965).

[66] M. Kardar, Nucl. Phys. B 290, 582 (1987).

[67] Y. C. Zhang, Phys. Rev. B 42, 4897 (1990).

[68] B. Derrida and J. L. Lebowitz, Phys. Rev. Lett. 80, 209 (1998).

[69] J. Z. Imbrie and T. Spencer, J. Stat. Phys. 52, 609 (1988).

[70] J. Cook and B. Derrida, Europhys. Lett. 10, 195 (1989).

[71] M. R. Evans and B. Derrida, J. Stat. Phys. 69, 427 (1992). 